\title{
Sharing Variable Returns of Cooperation
}

\author{
Maurice Koster*
}

February 21, 2005

\begin{abstract}
A finite set of agents jointly undertake a project. Depending on the aggregate of individual agent characteristics the project runs losses or profits, which have to be shared. This paper adopts the mechanistic view and concentrates on devices that a contingent planner may use in order to share the net profits. The Moulin and Shenker (1994) representation theorem is used to show that additive mechanisms with the constant returns property relate 1 to 1 to rationing methods. Refinements are discussed dealing with monotonicity and equity properties that relate to the dispersion of shares. The second part introduces the notion of a consistent solution. Each rationing method induced by a consistent mechanism is consistent. If such mechanism is continuous as well, then the corresponding rationing method is parametric. Most prevalent mechanisms (average, serial, Shapley-Shubik) are consistent as member of the class of incremental mechanisms. Each interval consistent incremental mechanism is shown to be a composition of marginal mechanisms and the average mechanism. Immediately the average mechanism is the unique strongly consistent solution. Finally a characterization of mechanisms within the general class is discussed using super-additivity.
\end{abstract}

Keywords: cooperative production, cost sharing, mechanism design, investment, (parametric) rationing, core, additivity, monotonicity

JEL-Classification: C70, D63, D70

\section{Sharing variable returns}

The simplest model of distributive justice is that of the rationing model as discussed in Moulin (2000). As Moulin (2002) shows, this model is closely related to the more involved cost sharing model where the amount to be shared is the costs for production of the aggregate of the individual demands. Israelsen (1980) discusses a dual model where workers share the returns from a jointly owned production facility, given the individual efforts or inputs. Both type of models have in common that for a given set of agents $N=\{1,2, \ldots, n\}$ and a list of individual characteristics $q \in \mathbb{R}_{+}^{N}$ there is an amount $f\left(\sum_{i} q_{i}\right)$ to be shared.

\footnotetext{
${ }^{*}$ Department of Quantitative Economics, Faculty of Economics and Econometrics, University of Amsterdam. E-mail: m.a.l.koster@uva.nl, phone: ++31-205254226.
} 
The distinction between the models is the way that the size of a share of $f\left(\sum_{i} q_{i}\right)$ is perceived: higher cost shares are depreciated, whereas higher production shares are appreciated. This paper abstracts from the above interpretations and considers the more general (variable) profit sharing problem where each agent $i$ is endowed with some input characteristic $q_{i}$ which in turn affects the total profits of the project via aggregation of the characteristics. This means that the profits of the project are described by a function $f$ that relates size of aggregate input characteristics to the degree of profitability of the project.

Apart from the standard cost sharing and production models, there are numerous other situations that fit into this model.

Investment The scale of many enterprizes may demand for several investors. Then its successfulness will amongst others - depend on the level of total investments. Typically, the dependency of investments and net profits possibly go in two directions: under-investment may well result in bankruptcy or shrinking business whereas over-investment may lower average returns per invested unit.

Common pool problems Agents invest in harvesting. The total of investments affects both a production as well as a cost component.

Consumer organizations Consumers pool demands in order to get better prices. The cost savings are related to the size of the aggregate demands. Or consider the pooling of savings in order to get better rates at bank.

The central question in all these problems is what the dispersion of the individual shares of the profits should be. In many applications profits are shared proportional to the agent characteristics. As for instance on stock-markets where an agents' characteristic is the number of shares owned and dividends are paid per share. Though the idea of proportionality is probably the oldest and the most widely spread, the literature on distributive justice discusses and promotes other solutions as well.

The success and the desirability of a particular solution depends on the actual context of the problem. The search for the ideal solution to an instance of a profit sharing problem can in general not be answered and should be regarded as ambiguous. Nevertheless it may put forward principles of profit sharing in a more general context within the sphere of a variety of problems. This paper follows the approach of the axiomatic literature in that it discusses several structural properties and looks to characterize solutions satisfying them (see e.g. Thomson (1999)). Some properties prescribe a solution in special cases of "obvious" problems, whereas others express an invariance of the solution to certain changes in the parameters of the problem. In this respect the most prominent properties will be consistency, that pertains to variations of the set of agents among whom the distribution of profits must take place, and additivity which allows a benevolent planner to combine results for several instances of problems to solve the problem by a single instance.

\section{Overview of the results and relation to the literature}

The paper shows ways to generalize the standard production sharing model where it is the outputs that have to be shared by agents having equal access rights for the production technology relative to their individual investments such as labor. The technology is fully described by a production function that assigns to each level of input the maximal output that can thus be generated. Then given a profile of individual investments the corresponding produced output has be allocated. This paper generalizes this model by interpreting the production function as a profit function. These profit functions may be decreasing, increasing, attain positive as well as negative values. The negative values are interpreted as costs. The class of problems with non-decreasing profit functions are the production problems as in Israelsen (1982) and Moulin and Shenker 
(1992). Problems with non-increasing profit functions are the cost sharing problems as in Moulin (2002). The same setup is chosen to address the problem of natural monopoly in Sharkey (1982) and Baumol et al. (1982). This paper considers solutions concepts to the cooperative profit sharing problems, i.e. devices that assign to each instance of a cooperative profit sharing problem a set of allocations.

Section 3 provides the basic setup for profits sharing problems, as well as the notion of a solution. Numerous examples of solutions and mechanisms are provided. Special solutions are the mechanisms that correspond to singletons. The solutions in this paper are all direct generalizations from the cost sharing literature. Relevant examples in the mechanistic cost sharing literature include solutions like the average cost sharing mechanism, the Shapley-Shubik mechanism (Shubik (1962), Sudhölter (1998), Young (1985)), the serial mechanism (Moulin and Shenker $(1992,1994)$ ). Multi-valued solutions are defined as the cores or imputation sets of relevant cooperative transferable utility games, one of which is the stand-alone profit game that is inspired by the stand-alone cost game as in Young $(1985,1994)$.

Section 4 focusses on the relation between a specific class of mechanisms and the class of rationing methods. More specifically, it is shown that positive and additive profit sharing mechanisms that satisfy constant returns are characterized by monotonic and continuous rationing methods. This is an extension of the characterization result of Moulin and Shenker (1994). It is shown that though the above class may be considered rather small, the (huge) pessimistic core consists exactly of the solutions generated by it. Properties are discussed as to describe how the individual shares of profits are related. Like equal treatment of equals and profit- and loss monotonicity, which are variants of the ranking property in the cost sharing literature. Then using these properties a refinement of the generalized Moulin and Shenker (1994) theorem are derived. In addition, the average mechanism is the unique mechanism that satisfies a weak solidarity property - that of nullity - as well.

Section 5 introduces concepts of consistency for profit sharing solutions that describes invariance of solutions in a model with varying sets of agents. The proposed concepts are alternatives to those found in the cost sharing literature (see, e.g., Young (1985), Moulin and Shenker (1994) and Sudhölter (1998)). Each consistent mechanism in the class of mechanisms that is characterised in the previous section is represented by a family of consistent rationing methods as in Young (1987) and Moulin $(2000,2002)$. In addition, the counterpart of Theorem 1 of Young (1987) is that each family of rationing methods that defines a continuous, consistent and symmetric mechanism is parametric.

The class of incremental mechanisms is introduced that can be seen to generalize the notion of random order value of Weber (1988). It is the class that corresponds to piecewise linear rationing methods. Each mechanism that is represented by a family of consistent and piecewise linear rationing methods is consistent. As a result most of the prevalent additive mechanisms in the literature are consistent. Nevertheless, there are just a few that satisfy the stronger property of interval consistency, i.e., the mechanisms that combine the marginal mechanisms and the average mechanism. In other words, using the terminology of Moulin (2000) for rationing methods, the irreducible interval consistent incremental mechanisms are the average mechanism and the marginal mechanisms. This leads us to conclude that, basically, the average sharing mechanism is the unique mechanism that is both incremental and strongly consistent. For each profits sharing problem the solutions of the consistent incremental mechanisms are dense in the set of solutions generated by the class of additive mechanisms with the properties positivity and constant returns. The results for set-valued solutions are that the maximal consistent solution satisfying the bounds put forward by the pessimistic individual bounds is the pessimistic core.

Section 6 studies general (set) solutions and characterizes the mechanisms using a weakened form of additivity together with properties like non-emptiness and bounds. 


\section{The profit sharing problem and preliminaries}

Throughout this paper we will restrict ourselves to a fixed and finite group of agents $N=\{1,2, \ldots, n\}$. The group of agents is involved in a joint project. The successfulness of the project hinges upon a profile of agent characteristics. One may think of a profile of individual demands, as in cost sharing, or a profile of individual investments such as labor input or money. Each such a profile of agent characteristics, or inputs, is summarized by a vector $q \in \mathbb{R}_{+}^{N}$. It is assumed that the relation between the inputs and net profits or net returns of the project are summarized by a profit function $f: \mathbb{R}_{+} \rightarrow \mathbb{R} ; f\left(\sum_{i \in N} q_{i}\right)$ indicates the returns of the project in case of profile of inputs $q \in \mathbb{R}^{N}$. A special situation occurs when the aggregate level of the agent characteristics is zero. It will be assumed that then $f(0)=0$; nothing happens when the aggregate of agent characteristics is zero. In the cost sharing context this condition is mostly understood as the absence of fixed costs. In addition, it is assumed that a profit function is an absolutely continuous function of the aggregate input variable ${ }^{1}$. In particular this means that such a function is differentiable almost everywhere where. Then for any profit function $f$ we will take $f^{\prime}: \mathbb{R}_{+} \rightarrow \mathbb{R}$ to denote the function that equals the derivative of $f$, if it exists, and that takes the value 0 otherwise. This function will be referred to as the marginal profit function $f^{\prime}$. By absolute continuity each marginal profit function $f^{\prime}$ is Lebesgue integrable and we may express total profits for at input level $y$ in terms of the marginal profit function $f^{\prime}$, since for all $t \in \mathbb{R}_{+}$we have

$$
f(t)=\int_{0}^{t} f^{\prime}(s) \mathrm{d} s .^{2}
$$

The set of all profit functions is denoted by $\mathcal{F}$. Special functions are the zero function and the identity defined by $f_{z}(y)=0$ and $f_{\text {id }}(y)=y$ for all $y \in \mathbb{R}_{+}$respectively. We distinguish two special classes in $\mathcal{F}$, $\mathcal{F}_{-}$and $\mathcal{F}_{+}$, the classes of all non-increasing and non-decreasing elements in $\mathcal{F}$, respectively. An element in $\mathcal{F}_{-}$is also referred to as cost function, when negative profits are interpreted as costs. The classes $\mathcal{F}_{-}$and $\mathcal{F}_{+}$are dual in the sense that $f \in \mathcal{F}_{-} \Leftrightarrow-f \in \mathcal{F}_{+}$. Each element $f \in \mathcal{F}$ can be written as the difference of two elements of $\mathcal{F}_{+}$. In order to see this define $f^{+}, f^{-} \in \mathcal{F}_{+}$by

$$
\begin{aligned}
& f^{+}(y)=\int_{0}^{y} \max \left\{f^{\prime}(t), 0\right\} \mathrm{d} t \\
& f^{-}(y)=-\int_{0}^{y} \min \left\{f^{\prime}(t), 0\right\} \mathrm{d} t .
\end{aligned}
$$

Then $f=f^{+}-f^{-}$.

A profits sharing problem for the agents in $S \subseteq N$ is an ordered pair $(q, f)$ consisting of a profit function $f \in \mathcal{F}$ and a vector of inputs $q \in \mathbb{R}_{+}^{S}$, that summarizes the individual characteristics of the individual agents; $q_{i}$ is the characteristic of agent $i \in S$. The set of all profit sharing problems for $S$ is denoted $\mathcal{R}^{S}$; moreover put $\mathcal{R}:=\bigcup_{S \subseteq N} \mathcal{R}^{S}$. The subset of $\mathcal{R}^{S}$ of all profit sharing problems with $c \in \mathcal{F}_{+}$is denoted $\mathcal{R}_{+}^{S}$, and $\mathcal{R}_{+}:=\bigcup_{S \subseteq N} \mathcal{R}_{+}^{S}$. Similarly we define $\mathcal{R}_{-}^{S}$ and $\mathcal{R}_{-}$as the class of all profit sharing problems with profit function in $\mathcal{F}_{-}$; this is the class of all cost sharing problems.

\footnotetext{
${ }^{1}$ A function $f: \mathbb{R}_{+} \rightarrow \mathbb{R}$ is absolutely continuous if for all intervals $[a, b] \subset \mathbb{R}_{+}$and $\varepsilon>0$ there is a $\delta>0$ such that for every finite collection of pairwise disjoint intervals $\left(a_{k}, b_{k}\right) \subset[a, b], k=1,2, \ldots, n$ with $\sum_{k=1}^{n}\left(b_{k}-a_{k}\right)<\delta$, we have $\sum_{k=1}^{n}\left|f\left(b_{k}\right)-f\left(a_{k}\right)\right|<\varepsilon$.

${ }^{2}$ This follows by the Fundamental Theorem in Lebesgue (1904).
} 
For any vector $y \in \mathbb{R}^{S}$ and $S \subseteq N$ define $y(S)=\sum_{i \in S} y_{i}$; denote the unit simplex $\left\{y \in \mathbb{R}_{+}^{S} \mid y(S)=1\right\}$ by $\Delta(S)$. For $A \subseteq \mathbb{R}$ the indicator function $\mathbb{I}_{A}: \mathbb{R} \rightarrow\{0,1\}$ is defined by

$$
\mathbb{I}_{A}(t)= \begin{cases}1 & \text { if } t \in A \\ 0 & \text { else. }\end{cases}
$$

To each profit sharing problem $R=(q, f) \in \mathcal{R}$ we associate the pessimistic profit sharing problem $\left(q, f_{R}^{\text {P }}\right)$ where $f_{R}^{\text {P }} \in \mathcal{F}$ is defined by

$$
f_{R}^{\mathrm{P}}(y)= \begin{cases}\inf \left\{\int_{T} f^{\prime}(s) \mathrm{d} s \mid T \in \mathcal{B}([0, q(S)]) ; \lambda(T)=y\right\} & \text { if } y \in[0, q(S)], \\ f(y) & \text { if } y>q(S) .\end{cases}
$$

Here $\mathcal{B}([0, q(S)])$ stands for the Borel- $\sigma$-algebra on the interval $[0, q(S)]$ and $\lambda$ is the Lebesgue measure. So the pessimistic profits function $f_{R}^{\mathrm{P}}$ relates each level of aggregate inputs $y \in[0, d(S)]$ to a specific lower bound on profits that is the minimum of corresponding aggregate marginal profits on $[0, q(S)]$. In cost sharing terms this is what Tijs and Koster (1998) defined by the pessimistic cost function. ${ }^{3}$ It is easy to show that each such pessimistic profits function $f_{R}^{\mathrm{P}}$ is convex on the interval $[0, q(S)]$.

Given a profit sharing problem $(q, f) \in \mathcal{R}^{S}$, the focus is on ways to assign the total profits $f(q(S))$ for the collective of agents $S \subseteq N$. For $(q, f) \in \mathcal{R}^{S}$ an element $y \in \mathbb{R}^{S}$ with the property that $y(S)=f(q(S))$ is called a vector of profit shares or just share vector. A solution is a mapping $\Psi$ that assigns for each $S \subseteq N$ to each $(q, f) \in \mathcal{R}^{S}$ a subset $\Psi(q, f) \subseteq \mathbb{R}^{S}$ of share vectors. In addition a solution $\Psi$ is called mechanism if it is single-valued, i.e. the set $\Psi(q, f)$ consists of precisely one element for all profit sharing problems $(q, f) \in \mathcal{R}^{S}, S \subseteq N$. The class of all solutions and mechanisms, resp., is denoted $\mathcal{S}$ and $\mathcal{M}$. By $\mathcal{S}^{+}$we denote the class of solutions on $\mathcal{R}_{+}$, i.e., all mappings $\Psi$ that assigns for each $S \subseteq N$ and $(q, f)$ in $\mathcal{R}_{+}^{S}$ a subset $\Psi(q, f) \subseteq \mathcal{R}^{S}$ of share vectors. Then $\mathcal{M}^{+}:=\mathcal{M} \cap \mathcal{S}^{+}$. With slight abuse of notation we will write $\Psi(q, f)=x$ whenever $x$ is the unique element in $\Psi(q, f)$. The class of solutions and mechanisms, resp., with properties $P_{1}, P_{2}, \ldots, P_{k}$ is denoted $\mathcal{S}\left(P_{1}, P_{2}, \ldots, P_{k}\right)$ and $\mathcal{M}\left(P_{1}, P_{2}, \ldots, P_{k}\right)$. A solution $\Psi \in \mathcal{S}$ satisfies positivity (POS) if for all $f \in \mathcal{F}_{+}$and $x \in \Psi(q, f)$ it holds that $x \geq 0$. A solution is nonempty (NE) if $\Psi(q, f) \neq \varnothing$ for all profit sharing problems $(q, f)$. In particular $\mathcal{M} \subseteq \mathcal{S}(\mathrm{NE})$ and $\mathcal{M}^{+} \subseteq \mathcal{S}^{+}(\mathrm{NE})$. Now we will discuss the solutions that will be most prominent in this paper. The focus will be on a fixed problem $R=(q, f) \in \mathcal{R}^{S}$.

\section{Examples of multi-valued solutions}

The multi-valued solutions in this paper all stem from ideas in cooperative game theory applied to the cooperative stand alone game (see, e.g., Young $(1985,1994)$ ) and pessimistic game (Tijs and Koster (1998)).

- The most trivial (multi-valued) solution that comes to mind is the set of all share vectors, i.e. the set

$$
\mathcal{A}(q, f)=\left\{x \in \mathbb{R}^{S} \mid x(S)=f(q(S))\right\} .
$$

- The imputation set is defined by

$$
\mathcal{I}(q, f)=\left\{x \in \mathbb{R}^{S} \mid x(S)=f(q(S)), \forall i \in S, x_{i} \geq f\left(q_{i}\right)\right\} .
$$

\footnotetext{
${ }^{3}$ A generalization of the pessimistic cost sharing problem to heterogeneous cost sharing problems is in Koster (2000).
} 
Related is the pessimistic imputation set, i.e., the set defined by

$$
\mathcal{I}^{\mathrm{P}}(q, f)=\mathcal{I}\left(q, f_{R}^{\mathrm{P}}\right) .
$$

- The stand alone core (Young (1994)) is the set of all share vectors

$$
\mathcal{C}(q, f)=\left\{x \in \mathbb{R}^{S} \mid x(S)=f(q(S)), \forall Q \subset S, x(Q) \geq f(q(Q))\right\} .
$$

The related pessimistic core is defined by

$$
\mathcal{C}^{\mathrm{P}}(q, f)=\mathcal{C}\left(q, f_{R}^{\mathrm{P}}\right)
$$

\section{Examples of mechanisms}

The mechanisms below are direct generalizations of mechanisms that are better known from the cost sharing literature. For a reference see Moulin (2002).

- The average cost sharing mechanism $\mu^{\mathrm{AV}}$ determines the share vector

$$
\mu^{\mathrm{AV}}(q, f)= \begin{cases}0 & \text { if } q(S)=0 \\ \frac{f(q(S))}{q(S)} \cdot q & \text { otherwise. }\end{cases}
$$

As a mechanism on $\mathcal{R}_{-}$it is better known as the average cost sharing mechanism (see e.g. Moulin (2002)).

- Let $\Pi(S)$ be the set of all mappings $S \rightarrow\{1,2, \ldots,|S|\}$. For each $\sigma \in \Pi(S)$ we define for $q \in \mathbb{R}_{+}^{S}$ the numbers $q_{0}^{\sigma}, q_{1}^{\sigma}, \ldots, q_{|S|}^{\sigma}$ by $q_{j}^{\sigma}=\sum_{\ell \leq j} q_{\sigma(j)}$. Then for any $\sigma \in \Pi(S)$, the corresponding marginal mechanism $\mu^{\sigma} \in \mathcal{R}^{S}$ is defined by

$$
\mu_{i}^{\sigma}(q, f):=f\left(q_{\sigma^{-1}(i)}^{\sigma}\right)-f\left(q_{\sigma^{-1}(i)-1}^{\sigma}\right) .
$$

So for each sharing problem $\mu^{\sigma}(q, f)$ is the marginal vector with respect to the stand alone game (see Young (1985)). The Shapley-Shubik mechanism $\Phi$ averages all marginal or incremental share vectors, i.e., $\Phi(q, f)=\frac{1}{n !} \sum_{\sigma \in \Pi(N)} \mu^{\sigma}(q, f) .{ }^{4}$ Define the pessimistic marginal mechanism with respect to $\sigma \in \Pi(N)$ by

$$
\mu_{p}^{\sigma}(q, f):=\mu^{\sigma}\left(q, f_{R}^{\mathrm{P}}\right), \text { for all } R=(q, f) \in \mathcal{R}^{S} .
$$

The pessimistic Shapley-Shubik mechanism $\Phi^{\mathrm{P}}$ is defined by $\Phi^{\mathrm{P}}(q, f)=\Phi\left(q, f_{R}^{\mathrm{P}}\right)$. Weber (1988) discusses the class of random order values consisting of all mechanisms that are a convex combination of marginal mechanisms.

- For $q \in \mathbb{R}_{+}^{S}$ let $\sigma \in \Pi(S)$ be such that $q_{\sigma(i)} \leq q_{\sigma(j)} \Leftrightarrow i \leq j$. Define numbers $q_{0}^{*}, q_{1}^{*}, \ldots, q_{|S|}^{*}$ by $q_{0}^{*}=0$ and $q_{j}^{*}=\sum_{\ell \leq j-1} q_{\sigma(\ell)}+(|S|-j+1) q_{\sigma(j)}$. Then the serial mechanism $\mu^{\mathrm{SR}}$ (see, e.g., Moulin and Shenker (1992)) is defined by

$$
\mu_{i}^{\mathrm{SR}}(q, f)=\sum_{\ell \leq \sigma(i)} \frac{f\left(q_{\ell}^{*}\right)-f\left(q_{\ell-1}^{*}\right)}{|S|-\ell+1} \text { for all } f \in \mathcal{F}, i \in S .
$$

\footnotetext{
${ }^{4}$ It is the Shapley value for the stand alone transferable utility game.
} 


\section{Additive mechanisms and rationing methods}

Additivity is propagated in the cost sharing literature as an accounting convention stating that decomposing a cost sharing problem in several cost components should not alter the final solution to the problem. Instead of focussing on several separate cost sharing problems, it is the property of additivity that allows for a single operation of calculation of cost shares. The same interpretation can be adopted for general profit sharing problems in those cases where it makes sense to calculate the shares in two problems with the same profile of agent characteristics.

Additivity A mechanism $\mu$ satisfies ADD if

$$
\mu\left(q, f_{1}+f_{2}\right)=\mu\left(q, f_{1}\right)+\mu\left(q, f_{2}\right)
$$

for all input profiles $q$ and $f_{1}, f_{2} \in \mathcal{F}$.

Suppose the profit function $f$ is the additive compound of the functions $f_{1} \in \mathcal{F}_{+}$and $f_{2} \in \mathcal{F}_{-}$. Then $f$ can be interpreted as the net revenues in the enterprize with profit function $f_{1}$ and cost function $f_{2}$. In turn, additivity requires that costs and profits are treated symmetrically.

Examples of additive mechanisms are numerous, amongst them we find $\mu^{\mathrm{AV}}, \mu^{\sigma}, \mu^{\mathrm{SR}}$ and $\Phi$. In this respect I like to point at the excellent overview on additive cost sharing in Moulin (2002). In particular in this work the power of the additivity as a mathematical tool becomes apparent. ${ }^{5}$

If marginal profits is constant relative to the agent characteristic, then it is reasonable if not compelling to credit the individual agents the same fixed marginal returns per unit of the characteristic. Then in case the agent characteristic is demand as in cost sharing models, or labor as in production models, then this would prescribe the same price and return per unit for each of the agents. So, in linear cases where where no externalities are present this amounts to the following property:

Constant Returns $\Psi$ satisfies $\mathrm{CR}$ if $\Psi\left(q, f_{\vartheta}\right)=\vartheta q$ for all $\vartheta>0$.

All previously discussed solutions satisfy $\mathrm{CR}$, except for $\mathcal{A}$. In particular, the variety of mechanisms in the class $\mathcal{M}(\mathrm{CR})$ is large, even if we would superimpose the additivity property. Nevertheless, within the class $\mathcal{M}^{+}$the set of all positive mechanisms with the properties ADD and CR is rather small in view of the Moulin and Shenker (1994) representation theorem. They show the 1-1 correspondence between rationing methods and additive cost sharing methods with CR property. Accordingly, each such cost sharing mechanism distributes marginal costs at each relevant level in a fixed ratio among the agents, and the share at each level is determined irrespective of the corresponding marginal costs. We will translate their result to fit the present model.

A rationing problem for coalition $S \subset N$ consists of a pair $(q, t) \in \mathbb{R}_{+}^{S} \times \mathbb{R}_{+}$such that $q(S) \geq t$. These are the most elementary problems in distributive justice and many types of problems can be modelled as

\footnotetext{
${ }^{5}$ Nevertheless, slowly a new branch develops in which non-additive methods are proposed and analyzed. See, e.g., Sprumont (1998), Tijs and Koster (1998), Koster (2001), Hougaard and Petersen (2001). The pessimistic methods above are examples of methods that are non-additive, and others are easily constructed by a composition $\mu \circ \varphi$ of an additive mechanism $\mu$ together with the non-additive transformation of the sharing problem $\varphi: R=(q, f) \mapsto\left(q, f_{R}^{\mathrm{P}}\right)$.Koster (2002) defines the pessimistic serial cost sharing mechanism that is a composite mapping with the serial cost sharing mechanism
} 
such; just to mention the literature on taxation problems (Young (1985)) and bankruptcy problems (Aumann and Maschler (1985)).

A rationing method $r$ for $S$ associates to any rationing problem $(q, t)$ a vector $r(q, t) \in[0, q]$ such that $\sum_{i \in S} r_{i}(q, t)=t$. A rationing method is monotonic whenever $t \leq t^{\prime}$ implies $r(q, t) \leq r\left(q, t^{\prime}\right)$ for all $t, t^{\prime}, q \in \mathbb{R}_{+}^{S}$. Then each such rationing method defines for all $q \in \mathbb{R}_{+}^{S}$ a monotonic (and continuous) path $t \mapsto r(q, t)$ from 0 to $q$. A rationing method is called piecewise linear if the path $t \mapsto r(q, t)$ is piecewise linear. Moulin and Shenker (1994) show the close relationship between rationing and cost sharing which can be translated in the terminology of this paper:

Theorem 4.1 Moulin and Shenker (1994)

Let $\mu \in \mathcal{M}^{+}(\mathrm{POS}, \mathrm{ADD}, \mathrm{CR})$. Then there is for each $S \subseteq N, q \in \mathbb{R}_{+}^{S}$ a monotonic rationing method $r(q, \cdot)$ such that

$$
\mu(q, f)=\int_{0}^{q(S)} f^{\prime}(t) \mathrm{d} r(q, t) \text { for all } f \in \mathcal{F}_{+} .
$$

Theorem 4.1 states that a mechanism in $\mathcal{M}^{+}(\mathrm{POS}, \mathrm{ADD}, \mathrm{CR})$ is fully characterized through its rationing system, i.e., a family of rationing methods $\pi:=\left\{r(q, \cdot) \mid q \in \mathbb{R}_{+}^{S}, S \subseteq N\right\}$, one for each input profile $q$. The mechanism corresponding to such family $\pi$ will be denoted $\mu^{\pi}$.

Remark In the above characterizations we can not leave out POS. Consider the mechanism $\mu$ that equals $\mu^{\mathrm{AV}}$ except for all profits sharing problems for the agents in $N=\{1,2\}$. In these cases put

$$
\left\{\begin{array}{l}
\mu_{1}(q, f)=\frac{1}{2} f\left(2 q_{1}\right), \text { and } \\
\mu_{2}(q, f)=f\left(q_{1}+q_{2}\right)-\frac{1}{2} f\left(2 q_{1}\right) .
\end{array}\right.
$$

Then $\mu$ is additive, satisfies CR, but not POS. Moreover, $\mu$ is not representable by a family of rationing methods, since $\mu$ assumes information about profits outside the interval $[0, q(N)]$, as opposed to all mechanisms representable as (9).

The above characterization result is easily extended to profits sharing mechanisms on $\mathcal{R}$.

Proposition 4.2 Each $\mu \in \mathcal{M}^{+}(\mathrm{ADD})$ uniquely extends to $\bar{\mu} \in \mathcal{M}(\mathrm{ADD})$.

Theorem $4.3 \mu \in \mathcal{M}(\mathrm{POS}, \mathrm{ADD}, \mathrm{CR})$ if and only if $\mu=\mu^{\pi}$ for some rationing family $\pi=\{r(q, \cdot) \mid q \in$ $\left.\mathbb{R}_{+}^{S}\right\}$.

Below we will focus on some refinements of Theorem 4.3. First of all, note that in our model, except for the labeling of the agents it is only their individual input that may influence a solution. Then, if two agents can not be distinguished for these characteristics then it is reasonable to demand that they should be treated equally by the solution.

Equal Treatment $\Psi \in \mathcal{S}$ satisfies ET if for all $x \in \Psi(q, f)$ it holds that $x_{i}=x_{j}$ whenever $q_{i}=q_{j}$.

ET is weaker than the anonymity property in Moulin and Shenker (1992). Together with the properties 
POS,ADD,CR, equal treatment implies that the rationing methods for agents with equal inputs are the same.

Proposition $4.4 \mu \in \mathcal{M}(\mathrm{POS}, \mathrm{ADD}, \mathrm{CR}, \mathrm{ET})$ if and only if

(i) $\mu$ is generated by a rationing family $\pi$, i.e., $\mu=\mu^{\pi}$,

(ii) for all $q \in \mathbb{R}_{+}^{S}$ with $q_{i}=q_{j}$ for some $i, j \in S, r(q, \cdot) \in \pi$, it holds $r_{i}(q, \cdot)=r_{j}(q, \cdot)$

Proof. POS,ADD,CR implies the functional representation as in Theorem 4.3. Now suppose that there is $q \in \mathbb{R}_{+}^{S}$ and $i, j \in S$ with $q_{i}=q_{j}$ but that $r_{i}(q, \cdot)=r_{j}(q, \cdot)$ does not hold. Then by continuity there is an interval $U \subset[0, q(S)]$ such that $r_{i}(q, \cdot)>r_{j}(q, \cdot)$ on $U$. Consider $f \in \mathcal{F}$ defined by

$$
f(y)=\int_{0}^{y} \mathbb{I}_{U}(t) d t \text { for all } y \in \mathbb{R}_{+} .
$$

Then

contradiction.

$$
\mu_{i}(q, f)=\int_{0}^{q(S)} \mathbb{I}_{U}(t) \mathrm{d} r_{i}(q, t)>\int_{0}^{d(S)} \mathbb{I}_{U}(t) \mathrm{d} r_{j}(q, t)=\mu_{j}(q, f),
$$

Profit Monotonicity $\Psi \in \mathcal{S}$ satisfies $\mathrm{PM}$ if for all $R=(q, f) \in \mathcal{R}_{+}$it holds for all $x \in \Psi(q, f)$ that $x_{i} \geq x_{j}$ if $q_{i} \geq q_{j}$.

Profit monotonicity assures that, apart from the interpretation of the agent characteristics, the larger the characteristic is the larger the gains. The next property formulates the dual, that when there are only losses from the project, the ones with the larger inputs suffer more.

Loss Monotonicity $\Psi \in \mathcal{S}$ satisfies LM if for all $R=(q, f) \in \mathcal{R}_{-}$it holds for all $x \in \Psi(q, f)$ that $x_{i} \geq x_{j}$ if $q_{i} \leq q_{j}$.

Both PM and LM can be considered weak: for all occurrences of mixed externality the axioms are even vacuous. And, moreover, still they do not affect flexibility in treating pure losses differently from profits in the sense that for $\mu \in \mathcal{M}(\mathrm{PM}, \mathrm{LM})$ and the dual problems $(d, f) \in \mathcal{R}_{+},(q,-f) \in \mathcal{R}_{-}$it need not hold that $\mu(q, f)=-\mu(q,-f)$. Nevertheless, together with ADD this degree of freedom is lost. The following shows that the dispersion of the shares in case of losses is the same for profits.

Proposition 4.5 Let $Y \in\{\mathrm{PM}, \mathrm{LM}\}$. Then $\mu \in \mathcal{M}(\mathrm{POS}, \mathrm{ADD}, \mathrm{CR}, Y)$ if and only if

(i) $\mu$ is generated by a rationing family $\pi$, i.e., $\mu=\mu^{\pi}$,

(ii) for all $q \in \mathbb{R}_{+}^{S}, i, j \in S, r(q, \cdot) \in \pi, q_{i} \geq q_{j} \Rightarrow r_{i}(q, \cdot) \geq r_{j}(q, \cdot)$

The proofs are similar to that of Proposition 4.4 and we omitted them for this reason. Notice that Proposition 4.5 implies that any mechanism $\mu \in \mathcal{M}(\mathrm{POS}, \mathrm{ADD}, \mathrm{CR}, Y)$ for $Y \in\{\mathrm{PM}, \mathrm{LM}\}$ satisfies ET. 
The representation of mechanisms by rationing families sees to shares of the same sign in case $f \in \mathcal{F}_{+}$ or $f \in \mathcal{F}_{-}$. A somewhat different property, but still related, is the following:

Null $\Psi \in \mathcal{S}$ satisfies NULL if for all $(q, f) \in \mathcal{R}^{S}$ with $f(q(S))=0$ it holds $\Psi(q, f)=\{0\}$.

Notice that NULL suggests to comprise all information in the profits function into a single point of evaluation as in case of $\mu^{\mathrm{AV}}$. Then on top of the characterizing properties in Theorem 4.3, by discarding all intermediate information about marginal revenues, it is strong enough to pin down the set of eligible candidates to a singleton.

Theorem 4.6 $\mathcal{M}(\mathrm{POS}, \mathrm{ADD}, \mathrm{CR}, \mathrm{NULL})=\left\{\mu^{\mathrm{AV}}\right\}$.

Proof. Take $\mu \in \mathcal{M}$ (POS, ADD, CR, NULL). Then according to Theorem 4.3 there is a rationing family $\pi=\left\{r(q, \cdot) \mid q \in \mathbb{R}_{+}^{S}, S \subseteq N\right\}$ such that $\mu=\mu^{\pi}$. Now suppose that there is $S \subseteq N, q \in \mathbb{R}_{+}^{S} \backslash\{0\}$ such that not $r(q, t)=t q / q(S)$ for all $t$. By continuity of $t \mapsto r_{i}(q, t)$ there are $i \in S$ and intervals $U, V$ in $[0, q(S)]$ such that

$$
\left\{\begin{array}{l}
r_{i}(q, t)<t q_{i} / q(S) \text { for all } t \in U, \text { and } \\
r_{i}(q, t)>t q_{i} / q(S) \text { for all } t \in V .
\end{array}\right.
$$

In particular we may choose $U, V$ so that $0<\lambda(U)=2 \lambda(V)$. Define $f \in \mathcal{F}$ by

$$
f(y)=\int_{0}^{y}\left(2 \cdot \mathbb{I}_{V}(t)-\mathbb{I}_{U}(t)\right) \mathrm{d} t \text { for all } y \in \mathbb{R}_{+} .
$$

Then

$$
\begin{aligned}
\mu_{i}(q, f) & =\int_{0}^{q(S)} f^{\prime}(t) \mathrm{d} r_{i}(q, t)=2 \int_{V} \mathrm{~d} r_{i}(q, t)-\int_{U} \mathrm{~d} r_{i}(q, t) \\
& >2 \lambda(V) \cdot q_{i} / q(S)-\lambda(U) \cdot q_{i} / q(S)=0 .
\end{aligned}
$$

But also $f(q(S))=0$ and thus, by NULL, $\mu_{i}(q, f)=0$ which gives the desired contradiction.

The above theorems makes clear that additivity is strong as a characterizing property since the combination with other reasonably weak properties leaves no other choice than the mechanisms induced by some rationing family. Nevertheless, below we will show that despite the fact that this class of mechanisms might be perceived as small, the induced range of profits shares is as large as the bounds of the pessimistic core prescribe. The following result depends on two lemmata that are put in the Appendix.

Theorem 4.7 For any $R=(q, f) \in \mathcal{R}^{S}$ it holds

$$
\mathcal{C}^{\mathrm{P}}(q, f)=\{\mu(q, f) \mid \mu \in \mathcal{M}(\mathrm{POS}, \mathrm{ADD}, \mathrm{CR})\} .
$$

\section{Consistency}

Most of the properties discussed earlier describe relations between shares of different instances of profit sharing problems. For example, ADD links profits sharing problems with different profits functions and 
ET connects outcomes on the basis of input profiles. This section focusses on the remaining element of the profits sharing problem, that is the set of agents. In the economic theory there has been enormous interest in consistency of ideas, that allows us to use similar principles with varying populations of interacting agents. The basic idea is that a fair solution should still be perceived so when viewed by any subgroup of agents. Young $(1985, \mathrm{p} 19)$ states that a solution should be stable such that 'no subgroup should want to "re-contract". This kind of stability that is usually referred to as consistency is studied in various fields of economics. Moreover in the more recent literature Friedman (1997) considers consistency properties in heterogeneous cost sharing problems. Davis and Maschler (1965) and Hart and Mas-Colell (1989) introduce the property to the field of cooperative games, whereas Sudhölter uses these ideas for solutions on cooperative cost sharing games. Other examples are Young (1985b) on taxation problems and Moulin (1987) on a model of surplus sharing. ${ }^{6}$

For rationing problems ideas of consistency are intuitive. A rationing method $r$ is called consistent if it holds for all rationing problems $(q, x)$ and $j \in S$ that

$$
r_{S \backslash\{j\}}(q, x)=r\left(q_{S \backslash\{j\}}, x-r_{j}(q, x)\right) .
$$

Hence, consistency states that upon removing an agent from the cooperative $S$, and taking all the resources that are allocated to this agent, renewed allocation of the remaining pieces within the reduced society does not make a difference as long as $r$ is used. As Moulin (2002) puts it

"changing the status of an agent from "active participant" to "passive expense of resources" does not alter the overall distribution; removing one agent and his share of resources is of no consequence to other agents.

Thus Consistency is a decomposition property with respect to changes in the set of relevant agents.'

A family of rationing methods is called consistent if each of its members is. Moulin (2002) asks for a concept of consistency according to which there is a one-to-one relationship to consistent mechanisms and an (partial) answer is given below.

Suppose that the group of agents $N$ face a profits sharing problem $(q, f) \in \mathcal{R}^{N}$ and that the mechanism $\mu$ is used to calculate the individual shares. One of the agents in $N$, say $i$, leaves the problem by taking his share $\mu_{i}(q, f)$. Suppose that now the remaining collective of agents $N \backslash\{i\}$ may renegotiate about the problem of sharing the leftovers. Basically there are two options, one is to stick to the old solution and the other is to recalculate the shares according to $\mu$. Then $\mu$ is called consistent if it leaves the vector of shares unchanged. This means that agents should not worry about renegotiating since basically nothing will help them to improve upon the status quo. But the crucial point here is that in order to be able to apply a solution like $\mu$ again, first there has to be a clear understanding what is actually the new profits sharing problem. This amounts to a translation of the original problem into a reduced problem $(\bar{q}, \bar{f}) \in \mathcal{R}^{N \backslash\{i\}}$ where the pre-paid amount $\mu_{i}(q, f)$ is taken into account. Although it seems fairly reasonable to use $\bar{q}=q_{N \backslash\{i\}}$ as the new profile of inputs, it is harder to get consensus about the new profits function. This is illustrated in the cost sharing literature where several reductions are proposed. Examples of studies of consistent solution concepts for cost sharing problems are Moulin and Shenker (1994), Kolpin (1994), and Sudhölter (1998). These studies learn that the above approach toward consistency is too stringent as the type of reduction almost seems to define the consistent mechanism. Although the models of profit sharing and rationing are closely related via the above characterization results, there seems to be more than only a technical problem

\footnotetext{
${ }^{6}$ Thompson (1996) gives a general overview on the use of consistency in the economic theory, and cost sharing problems in particular.
} 
of specifying what consistency means in either models. Whereas there is a natural and very intuitive meaning in the rationing model, a clear understanding for the profit sharing model is absent.

In this paper I will suggest a way out of this problem as follows. As it is not clear which reduction fits the practical situation best, the proposal is to include all problems that could possibly serve as a proper description of a reduction. Then in general a solution will be called consistent if any sharing vector in the original solution is still available for the remaining agents in the profits sharing problem with respect to some reduction. Crucial again is what should count as an admissible reduction. I will highlight the approach in this paper by an example.

\section{Example}

Consider a production facility jointly owned by three agents $N=\{1,2,3\}$. The agents characteristic is the amount of labor in hours they offer as input and assume that the production technology is described by the function $p(y)=y^{2}$; that is, if aggregate labor input is $y$ hours then the total of output generated is $y^{2}$. Suppose that the inputs of the agents is given by the profile $q=(1,2,3)$ and that $\mu^{\mathrm{AV}}$ is used as mechanism to share the output. Then the resulting share vector is $\mu^{\mathrm{AV}}(q, p)=(6,12,18)$. Suppose that only one agent can be at work at a time so that they work in shifts. If in the following an interval $[v, w]$ indicates a shift from time $v$ to $w$ then consider the following schedule, $s_{i}$ denotes the time that agent $i$ is active:

$$
s_{1}=\left[0, \frac{1}{2}\right) \cup\left[5 \frac{1}{2}, 6\right], s_{2}=[2,4), s_{3}=\left[\frac{1}{2}, 2\right) \cup\left[4,5 \frac{1}{2}\right) .
$$

This means that first agent 1 works for 30 minutes, then agent 3 for 90 minutes, etcetera. Check that according to this scheme the average profits reflect exactly the marginal productivity of the agents. Now suppose it is agent 2 that leaves the problem. One possible reduction is the one where the full information about marginal production at hours where the remaining agents 1 and 3 were active is kept intact. In other words, if the time that agent 2 worked are "cut out" then a possible reduced problem is $\left(q_{N \backslash\{2\}}, h^{*}\right)$ where $h^{*} \in \mathcal{F}$ is given by

$$
h^{*}(y)= \begin{cases}h(y) & \text { if } y \leq 2 \\ h(y+2)-p(4) & \text { if } y>2 .\end{cases}
$$

Similarly, if agent 1 would leave then this would result in the reduction $\left(q_{N \backslash\{1\}}, h^{* *}\right)$ with

$$
h^{* *}(y)= \begin{cases}h\left(y+\frac{1}{2}\right)-h\left(\frac{1}{2}\right) & \text { if } 0 \leq y \leq 5 \\ h(y+1)-h(6)+h\left(5 \frac{1}{2}\right)-h\left(\frac{1}{2}\right) & \text { if } y>5\end{cases}
$$

The above schemes are not at all unique in the sense that other schedules exist that correspond to the marginal productivity of the agents as well. Just consider the following schedule:

$$
s_{1}^{*}=\left[2 \frac{1}{2}, 3 \frac{1}{2}\right), s_{2}^{*}=[0,1) \cup[5,6], s_{3}^{*}=\left[1,2 \frac{1}{2}\right) \cup\left[3 \frac{1}{2}, 6\right) .
$$

Then if we want to cancel out the levels at which the leaving agent was at work then this scheme would result in a different reduction than $\left(q^{*}, h^{*}\right)$ namely the reduction by agent 2 could as well be defined by 
$\left(q_{N \backslash\{2\}}, \bar{h}\right)$ with

$$
\bar{h}(y)= \begin{cases}h(y+1)-1 & \text { if } 0 \leq y \leq 4 \\ h(y+2)-12 & \text { if } y>4\end{cases}
$$

As in the above example a plausible reduction will address different levels of input to the leaving agent, such that both the aggregate of these levels equals the input characteristic and the corresponding marginal profits the share of the agent.

For any bounded set $U \in \mathcal{B}([0, \infty))$ and $y \in \mathbb{R}_{+}$define $U_{y} \subset[0, \infty)$ as the smallest interval containing $[0, y]$ such that $\lambda\left(U_{y} \backslash U\right)=y$. Then the reduced profits function with respect to $U, f_{U}$, is defined by

$$
f_{U}(y)=\int_{U_{y}} f^{\prime}(t) \mathbb{I}_{\mathbb{R}_{+} \backslash U}(t) \mathrm{d} t \text {, for all } y \in \mathbb{R}_{+} .
$$

So $f_{U} \in \mathcal{F}$ takes for each input level $y$ the total of marginal returns of the first $y$ levels outside $U$. For any $R=(q, f) \in \mathcal{R}^{S}$ and $i \in S$ define $\mathcal{Q}(\Psi, R, i)$ as the set of all $T \in \mathcal{B}([0, q(S)])$ such that

(i) $\lambda(T)=q_{i}$

(ii) $\int_{T} f^{\prime}(s) \mathrm{d} s \in \Psi_{i}(q, f)$.

Then $T \in \mathcal{Q}(\Psi, R, i)$ can be interpreted as a set of input levels that simultaneously represent agent $i$ 's individual input (condition $(i)$ ) and his share of profits (condition (ii)).

A solution $\Psi$ is consistent if each restriction of a share vector in the original solution is available for some reduced profits sharing problem. Then if this holds for all reductions, $\Psi$ is called strongly consistent. Formally:

Consistency $(\mathrm{CO})$

For all $R=(q, f) \in \mathcal{R}^{S}, i \in S$, and $y \in \Psi(R)$ there exists $U \in Q(\Psi, R, i)$ such that $y_{S \backslash\{i\}} \in$ $\Psi\left(q_{S \backslash\{i\}}, f_{U}\right)$.

\section{Strong Consistency ( $\mathrm{SCO})$}

For all $R=(q, f) \in \mathcal{R}^{S}, i \in S, y \in \Psi(R)$, it holds $Q(\Psi, R, i) \neq \varnothing$, and $U \in Q(\Psi, R, i) \Longrightarrow y_{S \backslash\{i\}} \in$ $\Psi\left(q_{S \backslash\{i\}}, f_{U}\right)$.

Below we focus on a version of consistency that is weaker than SCO but stronger than CO. It requires consistency in general and consistency with respect to any reduction by intervals:

Interval Consistency (ICO)

$\Psi \in \mathcal{S}(\mathrm{CO})$ and for all $R=(q, f) \in \mathcal{R}^{S}, i \in S, y \in \Psi(R)$ it holds that $I=\left[t, t+q_{i}\right] \in Q(\Psi, R, i)$ implie that $y_{S \backslash\{i\}} \in \Psi\left(q_{S \backslash\{i\}}, f_{I}\right)$. Note that $\mathcal{S}(\mathrm{SCO}) \subset \mathcal{S}(\mathrm{ICO}) \subset \mathcal{S}(\mathrm{CO})$.

So the above notions of consistency require that any eligible share vector in the solution should be feasible by a proper choice of the reduction. Consistency requires at least one suitable reduction whereas strong consistency requires invariance of the solution with respect to any reduction that fits. Analogously, the notion of (strong) interval consistency restrict the attention to specific reductions, i.e., those induced by 
intervals.

The gap between these notions of consistency and others found in the cost sharing literature is eminent. The approaches towards consistency as in Moulin and Shenker (1994), Kolpin (1994), and Sudhölter (1998) and Kolpin (1994) are all alike in the sense that the reduction consists of the reduced demand profile together with a truncated cost function. For instance, consider consistency as in Moulin and Shenker (1994), where for $(q, c) \in \mathcal{R}_{-}^{S}$ and $i \in S$ and cost sharing mechanisms $\Psi$ the reduced cost function is determined by $\bar{f}(y)=\max \left\{c(y)-\Psi_{i}(q, c), 0\right\}$ learns that ${ }^{7}\left(q_{S \backslash\{i\}}, \bar{c}\right)$ would only be a reduction in the above sense if $\Psi_{i}(q, c)=c\left(q_{i}\right)$. The problem with the approach in Sudhölter (1998) is that the Davis-Maschler and Hart-Mas-Collell reduced games do not correspond to reduced profits sharing problems anymore since the condition $f(0)=0$ is in general not satisfied. However, the new proposal requires a more subtle approach in that the size of the truncation should counterbalance the share of the agent that goes away. The next example shows that the proposed consistency is rather weak and it leaves space for most of the discussed solution concepts.

Example 5.1 The marginal mechanisms $\mu^{\sigma}$ are consistent but not strongly consistent. Consider $R=$ $(q, f) \in \mathcal{R}^{S}$. First observe that clearly $T_{i}:=\left[q_{\sigma^{-1}(i)-1^{\prime}}^{\sigma} q_{\sigma^{-1}(i)}^{\sigma}\right] \in \mathcal{Q}\left(\mu^{\sigma}, R, i\right)$ for all $i \in S$. Then $\left(q_{S \backslash\{i\}}, f_{T_{i}}\right)$ is a reduced profits sharing problem with respect to $i$. Moreover, according to $\mu^{\sigma}$ the same ordering of the remaining agents is used to calculate the individual shares in the reduced profits sharing problem. Then the immediate consequence is that nothing changes for the agents $k$ with $\sigma(k)<\sigma(i)$. Now consider an agent $k$ with $\sigma(k)>\sigma(i)$. Then using the notation $q_{j}^{t}:=\sum_{j \in S \backslash\{i\}: \sigma(j) \leq t} q_{j}$ we get

$$
\begin{aligned}
\mu_{k}^{\sigma}\left(q_{S \backslash\{i\}}, f_{T_{i}}\right) & =f_{T_{i}}\left(q_{j}^{\sigma(k)}\right)-f_{T_{i}}\left(q_{j}^{\sigma(k)-1}\right)= \\
& =f\left(q_{i}+q_{j}^{\sigma(k)}\right)-f\left(q_{i}+q_{j}^{\sigma(k)-1}\right)= \\
& =f\left(\sum_{j \in S: \sigma(j) \leq \sigma(k)} q_{j}\right)-f\left(\sum_{j \in S: \sigma(j)<\sigma(k)} q_{j}\right)=x_{k}^{\sigma}(q, f) .
\end{aligned}
$$

This means that $\mu^{\sigma}$ is consistent (also in the sense of Moulin and Shenker (1994)). Nevertheless, $\mu^{\sigma}$ is not strongly consistent. To see this consider the problem $R=(q, f) \in \mathcal{R}^{\{1,2,3\}}$ with $f(y)=\int_{[0, y]} \mathbb{I}_{[0,1] \cup[2,3]}(t) \mathrm{d} t$ and $q=(1,1,1)$. Suppose that $\sigma(i)=i$ for all $i \in\{1,2,3\}$. Then $\mu^{\sigma}(q, f)=(1,0,1)$. Then $[2,3] \in \mathcal{Q}\left(\mu^{\sigma}, R, 1\right)$, but $x_{2}^{\sigma}\left(q_{\{2,3\}}, f_{[2,3]}\right)=1 \neq \mu_{2}^{\sigma}(q, f)$. In a similar way one may prove that the pessimistic marginal sharing mechanisms $\mu_{p}^{\sigma}$ are consistent but not strongly so.

Theorem 5.2 If $\mu=\mu^{\pi}$ is consistent, then $\pi$ is a consistent rationing family.

\footnotetext{
${ }^{7}$ Moulin and Shenker (1994) also discuss a restricted type of consistency, namely consistency restricted to only the smallest agents. It is used as a characterizing property for the serial cost sharing mechanism (Moulin and Shenker (1992)). Kolpin (1994) generalizes this idea through baseline consistency to the heterogeneous goods cost sharing model.
} 
Proof. Define $\Gamma_{t}: \mathbb{R} \rightarrow \mathbb{R}_{+}$by $\Gamma_{t}(y)=\min \{y, t\}$ for all $y \in \mathbb{R}_{+}$. Then for all $x \in[0, q(S)]$

$$
\mu\left(q, \Gamma_{x}\right)=\int_{0}^{q(S)} \Gamma_{x}^{\prime}(t) \mathrm{d} r(q, t)=r(q, x) .
$$

Moreover, for each $j \in S$ we have for $R=\left(q, \Gamma_{x}\right),\left[x-\mu_{j}\left(q, \Gamma_{x}\right), x\right]=\left[x-r_{j}(q, x), x\right] \in Q(\mu, R, j)$. But then it holds by $\mathrm{CO}$ for all $x \in[0, q(S)]$ that

$$
r_{S \backslash\{j\}}(q, x)=\mu_{S \backslash\{j\}}\left(q, \Gamma_{x}\right)=\mu\left(q_{S \backslash\{j\}}, \Gamma_{x-r_{j}(q, x)}\right)=r\left(q_{S \backslash\{j\}}, x-r_{j}(q, x)\right) .
$$

The theory of distributive justice is often concerned with stable allocation schemes that are robust against small changes in the parameters that define the allocation problem at hand. In the rationing theory this stability is formulated through continuity. A rationing method $r$ is called continuous if it is jointly continuous in both arguments, i.e., $(q, t) \mapsto r(q, t)$ is continuous for all rationing problems $(q, t)$.

Similarly, a mechanism will be called continuous if small changes in both the profit function and agent characteristics do not cause huge relative differences in the corresponding shares. More specifically:

CONT Mechanism $\mu$ is continuous if for each $S \subseteq N$ the mapping $\mathbb{R}_{+}^{S} \times \mathbb{R}_{+}$defined by $(q, t) \mapsto \mu\left(q, \Gamma_{t}\right)$ is continuous.

Remark Notice that the notion can be considered weak for the reason that continuity encompasses small changes in base functions $\Gamma_{t}$ only. In order to avoid the hybrid character of CONT one may consider the replacement by the following properties that sees to a clear-cut distinction between continuity aspects of profits and agent characteristics:

Profit-continuity For each $S \subseteq N$ and $q \in \mathbb{R}_{+}^{S}$ the mapping $\mathbb{R}_{+} \rightarrow \mathbb{R}_{+}^{S}$ defined by $t \mapsto \mu\left(q, \Gamma_{t}\right)$ is continuous.

Input-continuity For each $S \subseteq N$ the mapping $\mathbb{R}_{+}^{S} \rightarrow \mathbb{R}_{+}^{S}$ defined by $q \mapsto \mu\left(q, \Gamma_{t}\right)$ is continuous on $\{q \mid q(S) \leq t\}$.

Then a mechanism that is both profit- as well as input-continuous is continuous.

Young (1987) defines the rich class of parametric rationing methods that contains numerous classic rationing methods like the proportional method and the uniform gains and uniform losses method.

Let $f: D \rightarrow \mathbb{R}$ be a real-valued function where $D \subset \mathbb{R}^{2}$ is a set in $\mathbb{R}_{+} \times[0, \Lambda]$ for some $\Lambda \in \mathbb{R}_{+} \cup\{\infty\}$. It is assumed that for any $(z, \lambda) \in D$ it holds that $f(z, 0)=0, f(z, \Lambda)=z$ and $\lambda \mapsto f(z, \lambda)$ is nondecreasing and continuous. Then for such an $f$ there is a unique rationing method $r$ such that

$$
r_{i}(x, t)=f\left(x_{i}, \lambda\right) \text { where } \lambda \text { is a solution of } \sum_{i \in S} f\left(x_{i}, \lambda\right)=t .
$$

This $r$ is then called the parametric rationing method for $f$. It is easily seen that such a method is consistent. ${ }^{8}$ Young (1987) characterizes the parametric methods as the methods that satisfy equal treatment, consistency and continuity, a result that is useful in the present framework of mechanisms as well.

\footnotetext{
${ }^{8}$ The class of parametric rationing methods is rich and contains for example the proportional rationing method, uniform gains and uniform losses methods. See for further references Moulin (2002).
} 
Lemma 5.3 If $\mu \in \mathcal{M}(\mathrm{CR}, \mathrm{ADD}, \mathrm{POS})$ is continuous then $\mu=\mu^{\pi}$ and $\pi$ is a family of continuous rationing methods.

Proof. Consider $\mu=\mu^{\pi} \in \mathcal{M}$. Then without loss of generality it may be assumed that $\pi$ consists of the rationing methods defined by

$$
r(q, t):=\mu\left(q, \Gamma_{t}\right) .
$$

Then clearly continuity of $\mu$ implies continuity of the method $r$.

Moreover, he proves the result that this class consists precisely of the methods that satisfy equal treatment of equals, continuity and consistency. This will be of use to characterize mechanisms. First I will introduce parametric rationing methods formally.

Theorem 5.4 Any $\mu \in \mathcal{M}(\mathrm{POS}, \mathrm{ADD}, \mathrm{CR}, \mathrm{CO}, \mathrm{ET}, \mathrm{CONT})$ is represented by a parametric rationing family.

Proof. By Proposition 4.4 it follows that the rationing family satisfies equal treatment. Then by Theorem 1 in Young (1987) and Lemma 5.3 the result obtains.

Proving results the other way around is more complex. Till this very moment I am not sure whether each consistent rationing method induces a consistent profit sharing mechanism. Nevertheless, below I will prove that consistency is transferred smoothly between the models of rationing and profit sharing as far as the incremental mechanisms and piecewise linear rationing methods are concerned.

All of the earlier examples of additive mechanisms have in common that a finite number of intermediate levels of input determines the final solution, as the profits increments of two consecutive levels is split amongst the agents in a fixed ratio. Recall the random order values in this respect, by which shares are determined on basis of the intermediate input levels of coalitional aggregate inputs. Here I will discuss a more general class of mechanisms, each of one splits increments related to other intermediate levels of input as well.

A mechanism $\mu$ is called an incremental mechanism if for each $q \in \mathbb{R}_{+}^{S}$ there is an integer $k \in \mathbb{N}$, vectors $\alpha_{1}, \alpha_{2}, \ldots, \alpha_{k} \in \Delta(S)$ and $x \in \mathbb{R}_{+}^{k+1}$ such that

$$
\mu(q, f)=\sum_{\ell=1}^{k} \alpha_{\ell}\left(f\left(x_{\ell}\right)-f\left(x_{\ell-1}\right)\right) \text { for all } f \in \mathcal{F},
$$

and where $0=x_{0} \leq x_{1} \leq \ldots \leq x_{k}=q(S)$. Then each such mechanism $\mu$ satisfies CR if only

$$
\sum_{\ell=1}^{k} \alpha_{\ell}\left(x_{\ell}-x_{\ell-1}\right)=q
$$

In that case $\mu \in \mathcal{M}^{+}(\mathrm{POS}, \mathrm{ADD}, \mathrm{CR})$ and the corresponding rationing family $\pi=\left\{r(q, \cdot) \mid q \in \mathbb{R}_{+}^{S}\right\}$ is determined by the piecewise linear mappings $t \mapsto r(q, t)$ such that

$$
\frac{\partial}{\partial t} r(q, t)=\alpha_{\ell} \text { for all } t \in\left(x_{\ell-1}, x_{\ell}\right)
$$


in case $x_{\ell} \neq x_{\ell-1}$. The set of all incremental mechanisms is denoted $\mathcal{M}^{I}$. Notice that $\mathcal{M}^{I} \subset \mathcal{M}^{+}$. Then $\mathcal{M}^{I}$ forms a generalization of the class of incremental methods and random order mechanisms as in Weber (1988).

\section{Examples}

- The average mechanism is an incremental mechanism with

$$
k=1, x_{k}=q(S), \alpha_{k}=\frac{q}{q(S)} \text { whenever } q(S)>0 .
$$

- For $q \in \mathbb{R}_{+}^{S}$ there is a permutation $\sigma \in \Pi(S)$ that such that $q_{\sigma(i)} \leq q_{\sigma(j)} \Leftrightarrow \sigma(i) \leq \sigma(j)$ for all $i, j \in S$. Then take $k=|S|$ and

$$
x_{\ell}=\sum_{p \leq \sigma^{-1}(\ell)-1} q_{\sigma(p)}+(|S|-\ell+1) q_{\sigma(\ell)} \quad \text { and } \quad \alpha_{\ell i}= \begin{cases}1 /(|S|-\ell+1) & \text { if } \sigma(i) \geq \ell, \\ 0 & \text { else. }\end{cases}
$$

The corresponding incremental mechanism is the serial mechanism.

- Each marginal mechanism $\mu^{\sigma}$ is incremental with $x_{\ell}, \alpha_{\ell}$ such that

$$
x_{\ell}=\sum_{p \leq \sigma^{-1}(\ell)} q_{\sigma(p)} \quad \text { and } \quad \alpha_{\ell i}=1 \Longleftrightarrow \sigma(i)=\ell .
$$

- Shapley-Shubik mechanism: the $x_{\ell}$ 's are generated by the aggregate investments $\{q(U) \mid U \subseteq S\}$ and

$$
\alpha_{\ell i}=1 /|S| ! \cdot \#\left\{\sigma \in \Pi(S) \mid\left[x_{\ell-1}, x_{\ell}\right] \subseteq\left[\sum_{j ; \sigma^{-1}(j)<i} q_{j} \sum_{j ; \sigma^{-1}(j) \leq i} q_{j}\right]\right\} .
$$

- An example of a mechanisms that is not incremental is $\mu_{p}^{\sigma}$.

The next result is that there is a one-to-one correspondence between the consistent incremental mechanisms and the consistent piecewise linear rationing methods.

Theorem $5.5 \mu \in \mathcal{M}^{I}(\mathrm{POS}, \mathrm{ADD}, \mathrm{CR})$ is consistent if and only if $\mu=\mu^{\pi}$ and $\pi$ is a consistent family of piecewise linear rationing methods.

One may easily verify that the rationing methods corresponding to the earlier mentioned incremental mechanisms are all consistent. Then an implication of Theorem 5.5 is that:

Corollary 5.6 The mechanisms $\mu^{\mathrm{AV}}, \mu^{\mathrm{SR}}, \mu^{\sigma}, \Phi$ are all consistent.

Moulin (2000) introduces the notion of a reducible rationing method. Basically, a rationing method is reducible if there is an ordered partition of the set of agents and for each element in the partition a (different) rationing method such that the final allocation can be determined in two steps: (1) for each element in 
the induced ordered partitioning of a coalition $S$ it is prescribed what units are available for this group of agents, (2) these units are shared among the corresponding agents according to the rationing method associated with this element in the partition. This two step procedure will be used for the profit sharing model as well. A mechanism will be called reducible if there is a non-trivial ordered partition $\mathcal{N}$ of $N$, such that profit shares may be calculated by different mechanisms to certain profit sharing problems induced by the ordered partition. Formally this procedure is as follows.

Consider an ordered partition $\mathcal{N}=(N(1), N(2), \ldots, N(\kappa))$ of $N$, where $\kappa$ is an integer smaller than $|N|$. Given $\mathcal{N}, R=(q, f) \in \mathcal{R}^{S}$ define for $k=1, \ldots, \kappa$ the profit function $f_{R}^{k} \in \mathcal{F}$ by

$$
f_{R}^{k}(y)=f\left(\sum_{\ell \leq k-1} q(N(\ell) \cap S)+y\right)-f\left(\sum_{\ell \leq k-1} q(N(\ell) \cap S)\right)
$$

for all $y \in \mathbb{R}_{+}$.

A mechanism $\mu \in \mathcal{M}$ is reducible if there is a non-trivial ordered partition $\mathcal{N}=(N(1), N(2), \ldots, N(\kappa))$ of $N$ together with mechanisms $\mu^{1}, \ldots, \mu^{\kappa} \in \mathcal{M}$ such that for each $(q, f) \in \mathcal{R}^{S}, i \in S \cap N(k)$ it holds

$$
\mu_{i}(q, f)=\mu_{i}^{k}\left(q_{S \cap N(k)}, f_{R}^{k}\right) .
$$

Then $\mu$ is also called the composition of the mechanisms $\mu^{1}, \ldots, \mu^{\kappa}$. So, as an analogue to the rationing model, here the ordered partition is used to address the different profit levels to the elements in the induced partition. The corresponding profit sharing problem consists of the relevant agent characteristics together with a profit function that carries all the information of the original problem of the relevant profit levels.

Theorem 5.7 $\mu \in \mathcal{M}^{I}(\mathrm{POS}, \mathrm{ADD}, \mathrm{CR})$ is interval consistent if and only if it is a composition of the average mechanism and marginal mechanisms.

Corollary 5.8 There is exactly one strongly consistent mechanism in $\mathcal{M}^{I}(\mathrm{POS}, \mathrm{ADD}, \mathrm{CR})$ and that is $\mu^{\mathrm{AV}}$.

Proof. First we will show that actually $\mu^{\mathrm{AV}}$ is strongly consistent. Consider a reduction of the problem $R=(q, f) \in \mathcal{R}^{S}$ with respect to agent $i \in S$, say $\left(q_{S \backslash\{i\}}, f_{T}\right)$, where $T \in \mathcal{Q}\left(\mu^{\mathrm{AV}}, R, i\right)$. Then

$$
\begin{aligned}
\mu^{\mathrm{AV}}\left(q_{S \backslash\{i\}}, f_{T}\right) & =\frac{q_{S \backslash\{i\}}}{q(S \backslash\{i\})} f_{T}(q(S \backslash\{i\})) \\
& =\frac{q_{S \backslash\{i\}}}{q(S \backslash\{i\})}\left\{f(q(S))-\frac{q_{i}}{q(S)} f(q(S))\right\} \\
& =\frac{q_{S \backslash\{i\}}}{q(S \backslash\{i\})} \frac{q(S \backslash\{i\})}{q(S)} f(q(S))=\mu_{S \backslash\{i\}}^{\mathrm{AV}}(q, f) .
\end{aligned}
$$

In order to show the uniqueness it is enough to point out that any reducible mechanism is not strongly consistent. Then Theorem 5.7 shows that only $\mu^{\mathrm{AV}}$ remains. This looks like exploiting the ideas of Example 5.1 . 
Remark In fact one may prove that the unique strongly consistent mechanism in $\mathcal{M}(\mathrm{POS}, \mathrm{ADD}, \mathrm{CR})$ is $\mu^{\mathrm{AV}}$. A proof is available upon request.

Corollary 5.9 Suppose $Y \in\{\mathrm{ET}, \mathrm{LM}, \mathrm{PM}\}$. The unique interval consistent incremental mechanism in $\mathcal{M}(\mathrm{POS}, \mathrm{ADD}, \mathrm{CR}, Y)$ is $\mu^{\mathrm{AV}}$.

These are applications from the properties of the rationing methods.

Note that the above proofs are also sound within the class of cost sharing problems, and may serve to identify the different cost sharing mechanisms.

Below there is a first characterization of the pessimistic core. It is the maximal consistent solution in the pessimistic imputation set.

Theorem 5.10 If $\Psi \in \mathcal{S}(\mathrm{CO})$ and $\Psi(R) \subseteq \mathcal{I}^{\mathrm{P}}(R)$ for all $R \in \mathcal{R}$, then $\Psi(R) \subseteq \mathcal{C}^{\mathrm{P}}(R)$ for all $R \in \mathcal{R}$.

The below result shows that in a way the consistent incremental mechanisms are dense in the set of all mechanisms represented by a family of rationing methods.

Lemma 5.11 Suppose that for a specific $\bar{q} \in \mathbb{R}_{+}^{U}, U \subseteq N$ there is a rationing method $\bar{r}$ such that $t \mapsto$ $\bar{r}(\bar{q}, t)$ is monotonic and piecewise linear. Then there is a rationing family $\pi=\left\{r(q, \cdot) \mid q \in \mathbb{R}_{+}^{S}, S \subseteq\right.$ $N\}$ such that $(i) r(\bar{q}, \cdot)=\bar{r}(\bar{q}, \cdot)$ and (ii) $\mu^{\pi} \in \mathcal{M}^{I}(\mathrm{POS}, \mathrm{ADD}, \mathrm{CR}, \mathrm{CO})$.

Proof. There are numbers $0=\bar{x}_{0}<\bar{x}_{1}<\ldots<\bar{x}_{k-1}<\bar{x}_{k}=\bar{q}(S)$ and $\bar{\alpha}_{1}, \ldots, \bar{\alpha}_{k} \in \Delta(U)$ such that

$$
\frac{\partial}{\partial t} \bar{r}(\bar{q}, t)=\bar{\alpha}_{\ell} \text { for all } t \in\left(\bar{x}_{\ell-1}, \bar{x}_{\ell}\right) \text {. }
$$

The definition of $r$ is completed as follows. Take $S \subseteq N$ and $q \in \mathbb{R}_{+}^{S}$. Define $U^{*}=\left\{j \in U \cap S \mid q_{j}=\bar{q}_{j}\right\}$. Determine $x_{1}, x_{2}, \ldots, x_{\mid U^{*}}$ by putting $x_{0}=0$ and for $\ell=1,2, \ldots, k$

$$
x_{\ell}=x_{\ell-1}+\left(1-\bar{\alpha}_{\ell}\left(U \backslash U^{*}\right)\right)\left(\tilde{x}_{\ell}-\tilde{x}_{\ell-1}\right) .
$$

Write $S \backslash U^{*}=\left\{i_{1}, i_{2}, \ldots, i_{\left|S \backslash U^{*}\right|}\right\}$ such that $i_{p}<i_{p+1}$ for all $p=1,2, \ldots,\left|S \backslash U^{*}\right|$. Moreover define $x_{k+\ell}=x_{k+\ell-1}+q_{i_{\ell}}$ for all $\ell=1,2, \ldots,\left|S \backslash U^{*}\right|$. In addition define $\alpha_{1}, \alpha_{2}, \ldots, \alpha_{k} \in \Delta(S)$ by

$$
\alpha_{\ell j}= \begin{cases}\frac{\bar{\alpha}_{\ell j}}{\bar{\alpha}_{\ell}\left(U^{*}\right)} & \text { if } j \in U^{*}, \\ 0 & \text { if } j \in S \backslash U^{*},\end{cases}
$$

Moreover $\alpha_{k+1}, \alpha_{k+1}, \ldots, \alpha_{k+\left|S \backslash U^{*}\right|}$ are vectors in $\Delta(S)$ such that $\alpha_{k+\ell}$ has all zeroes except for coordinate corresponding to agent $i_{\ell}$, which takes value 1 . Then, finally, define the monotonic and piecewise linear rationing method $r(q, \cdot)$ by

$$
\frac{\partial}{\partial t} r(q, t)=\alpha_{\ell} \text { for } \ell=1,2, \ldots, k+\left|S \backslash U^{*}\right|
$$


In this way we obtain a family of rationing methods $\pi=\left\{r(q, \cdot) \mid q \in \mathbb{R}_{+}^{S}, S \subseteq N\right\}$ that specifies a consistent incremental mechanism $\mu^{\pi}$.

Theorem 5.12 For each $\mu \in \mathcal{M}(\mathrm{POS}, \mathrm{ADD}, \mathrm{CR})$ and $q \in \mathbb{R}_{+}^{S}$ there is a sequence $\left\{\mu^{k}\right\}_{k=1,2, \ldots}$ of mechanisms in $\mathcal{M}^{I}(\mathrm{CO})$ such that $\mu(q, f)=\lim _{k \rightarrow \infty} \mu^{k}(q, f)$ for all $f \in \mathcal{F}$.

Proof. Let $\mu^{\bar{\pi}} \in \mathcal{M}(\mathrm{POS}, \mathrm{ADD}, \mathrm{CR})$, with $\bar{\pi}=\left\{\bar{r}(q, \cdot) \mid q \in \mathbb{R}_{+}^{S}, S \subseteq N\right\}$ the corresponding rationing family. Take $\bar{q} \in \mathbb{R}_{+}^{S}$. The mapping $t \mapsto \bar{r}(\bar{q}, t)$ is continuous. For each $n \in \mathbb{N}$ and $k=1,2, \ldots, 2^{n}$ define a number $t_{k}^{n}=k 2^{-n} \bar{q}(S)$. Define the piecewise linear rationing method $\bar{r}^{n}(\bar{q}, \cdot)$ by putting

$$
\frac{\partial}{\partial t} \bar{r}^{n}(\bar{q}, t)=\frac{\bar{r}\left(\bar{q}, t_{k}^{n}\right)-\bar{r}\left(\bar{q}, t_{k-1}^{n}\right)}{t_{k}^{n}-t_{k-1}^{n}} \text { for } t \in\left(t_{k-1}^{n}, t_{k}^{n}\right),
$$

for each $k=1,2, \ldots, 2^{n}$ and with $t_{0}^{n}=0$. Use Lemma 5.11 to construct a rationing family $\pi(n)=$ $\left\{r^{n}(q, \cdot) \mid q \in \mathbb{R}_{+}^{S}, S \subseteq N\right\}$ such that $r^{n}\left(\bar{q}_{1} \cdot\right)=\bar{r}^{n}\left(\bar{q}_{1} \cdot\right)$ and $\mu^{n}:=\mu^{\pi(n)}$ is consistent. Then since $r^{n}(q, \cdot) \rightarrow r(q, \cdot)$ uniformly we have $\mu^{n}(q, f) \rightarrow \mu(q, f)$ for $n \rightarrow \infty$, for each $f \in \mathcal{F}$.

\section{From solutions to mechanisms by super-additivity}

Below we will start with set solutions and state a corresponding generalized additivity property. It states that a contingent planner should profit from combining return sharing problems in the sense that the set of available options does not shrink by doing so. Compare this with additivity, that requires invariance with respect to such an operation. But first we need some extra notation. Given $S \subseteq N, X, Y \subset \mathbb{R}^{S}$ we define the direct sum of $X$ and $Y$ as the set $X \oplus Y:=\{z \mid \exists x \in X, y \in Y, z=x+y\}$ and the scalar product $\alpha X:=\{\alpha x \mid x \in X\}$.

Super-additivity A solution $\Psi$ is super-additive (SADD) if it holds that

$$
\Psi\left(q, f_{1}\right) \oplus \Psi\left(q, f_{2}\right) \subseteq \Psi\left(q, f_{1}+f_{2}\right),
$$

for all $f_{1}, f_{2} \in \mathcal{F}, q \in \mathbb{R}_{+}^{S}, S \subseteq N$.

Super-additivity states that combining several sharing problems into one does not limit the eligible set of shares as a solution. Of course, the class $\mathcal{S}(\mathrm{SADD})$ contains the set of all additive mechanisms $\mathcal{M}(\mathrm{ADD})$.

Zero property A solution $\Psi$ satisfies $\mathrm{Z}$ if $\Psi\left(q, f_{z}\right)=\{0\}$ for all $q \in \mathbb{R}_{+}^{S}, S \subseteq N$.

Proposition 6.1 $\mathcal{S}(\mathrm{NE}, \mathrm{SADD}, \mathrm{Z})=\mathcal{M}(\mathrm{ADD})$.

Proof. First we will show that $\mathcal{M}(\mathrm{ADD}) \subseteq \mathcal{S}(\mathrm{NE}, \mathrm{SADD}, \mathrm{Z})$. Take $\mu \in \mathcal{M}(\mathrm{ADD})$. Then for all $q$

$$
\mu\left(q, f_{z}\right)=\mu\left(q, f_{z}\right)+\mu\left(f_{z}\right)=2 \mu\left(f_{z}\right),
$$

hence $\mu\left(q, f_{z}\right)=0$. Take $\Psi \in \mathcal{S}(\mathrm{NE}, \mathrm{SADD}, \mathrm{Z})$. By SADD and $\mathrm{Z}$ we have for each $(d, f) \in \mathcal{R}$

$$
\Psi(q, f) \oplus \Psi(q,-f) \subseteq \Psi(q, f+(-f))=\Psi\left(q, f_{z}\right)=\{0\} .
$$


By NE both $\Psi(q, f)$ and $\Psi(q,-f)$ have at least one element. Hence it can not be the case that one of these sets has more than one element since that would mean that $\Psi(q, f) \oplus \Psi(q,-f)$ contains more than one element. Then $\Psi(q, f)=\{x\}, \Psi(q,-f)=\{y\}$ implies $x+y=0$ and $\Psi(q, f)=-\Psi(q,-f)$.

Boundedness A solution $\Psi$ satisfies $\mathrm{B}$ if for all for all $f \in \mathcal{F}$ it holds that $\Psi(q, f)$ is bounded in $\mathbb{R}^{S}$ for all investment profiles $q \in \mathbb{R}_{+}^{S}$. To be precise, for each $(q, f) \in \mathcal{R}^{S}$ there exists a $\gamma>0$ such that $\|\Psi(q, f)\|<\gamma$.

When a solution is unbounded, then there is at least one agent that is extremely exploited by another. Hence $\mathcal{B}$ is regarded a weak property, stating that finite differences in investment do not lead to arbitrary large differences in the shares of profit. It can be interpreted as a very weak incentive property for investing. Voluntary cooperation is more likely to occur if there are upper bounds on cost shares as well as lower bounds on return shares.

The properties NE, SADD, and Z are logically independent: $\mathcal{C}$ satisfies SADD and Z but not NE, $\mathcal{C}^{\mathrm{P}}$ satisfies NE, Z, but not SADD, whereas $\mathcal{A}$ satisfies both NE and SADD but not Z.

Proposition 6.2 $\mathcal{S}(\mathrm{NE}, \mathrm{SADD}, \mathrm{B})=\mathcal{M}(\mathrm{ADD})$.

Proof. It is sufficient to show that $\mathcal{S}(\mathrm{NE}, \mathrm{SADD}, \mathrm{B}) \subseteq \mathcal{S}(\mathrm{NE}, \mathrm{SADD}, \mathrm{Z})$. Suppose there exists

$$
\Psi \in \mathcal{S}(\mathrm{NE}, \mathrm{SADD}, \mathrm{B}) \backslash \mathcal{S}(\mathrm{NE}, \mathrm{SADD}, \mathrm{Z}) .
$$

Then there is $q \in \mathbb{R}_{+}^{S}$ and $x \in \mathbb{R}^{S} \backslash\{0\}$ such that $x \in \Psi\left(q, f_{z}\right)$. Then by induction and SADD it follows that $\left\{2^{k} x\right\}_{k \in \mathbb{N}}$, contradicting B.

\section{Logical independence}

- $\mathcal{C}$ satisfies SADD and B. It fails to be non-empty for some returns sharing problems. To see this, just consider any non-zero investment profile and a concave returns function. Then even the set of imputations is empty.

- $\mathcal{C}^{\mathrm{P}}$ satisfies B and NE, but does not satisfy SADD. Since for $q=(1,2)$ and $f_{1}(y)=\min \{y, 1\}$ en $f_{2}(y)=\max \{y-1,0\}$ we have

$$
\begin{aligned}
& \mathcal{C}^{\mathrm{P}}\left(q, f_{1}\right)=\left\{x \in \mathbb{R}^{2} \mid x_{1} \leq 1, x_{2} \leq 1, x_{1}+x_{2}=1\right\} \\
& \mathcal{C}^{\mathrm{P}}\left(q, f_{2}\right)=\left\{x \in \mathbb{R}^{2} \mid \begin{array}{l}
x_{1} \leq 1, x_{2} \leq 2, x_{1}+x_{2}=2 \\
x_{2}
\end{array}\right.
\end{aligned}
$$

In particular $(1,0) \in \mathcal{C}^{\mathrm{P}}\left(q, f_{1}\right)$ and $(1,1) \in \mathcal{C}^{\mathrm{P}}\left(q, f_{2}\right)$. Then $\mathcal{C}^{\mathrm{P}}$ cannot be sub-additive since $\mathcal{C}^{\mathrm{P}}\left(q, f_{1}+f_{2}\right)=\{q\}$ and $(1,0)+(1,1) \neq q$.

- The set of all share vectors $\mathcal{A}(d, f)=\left\{x \in \mathbb{R}^{S} \mid x(S)=f(d(S))\right\}$ for $(d, f) \in \mathcal{R}^{S}$ defines a solution that satisfies the properties SADD and NE, except B.

\section{References}

R. J. Aumann and M. Maschler, Game theoretic analysis of a bankruptcy problem from the talmud, Journal of Economic Theory 36 (1985), 195-213. 
W. Baumol, J. Panzar, R. Willig, and E. Bailey, Contestable markets and the theory of industry structure, revised ed., Harcourt Brace Jovanovich, San Diego, California, U.S.A., 1988.

M. Davis and M. Maschler, The kernel of cooperative games, Naval Research Logistics Quarterly 12 (1965), 223-259.

E.J. Friedman, Weak and strong consistency in additive cost sharing, Mimeo, Rutgers University, Newark, New Jersey, U.S.A., 1997.

S. Hart and A. Mas-Colell, Potential, value and consistency, Econometrica 57 (1989), 589-614.

J.L. Hougaard and L. Thorlund-Petersen, Mixed serial cost sharing, Mathematical Social Sciences 41 (2001), 51-68.

V. Kolpin, Coalition equitable cost sharing of multi-service facilities, Mimeo, University of Oregon, Eugene, Oregon, U.S.A., 1994.

M. Koster, Concave and convex serial cost sharing, Chapters in Game Theory (Dordrecht, The Netherlands) (P. Borm and H. Peters, eds.), Kluwer Academic Press, 2002.

H. Moulin, Equal or proportional division of a surplus, and other methods, International Journal of Game Theory 16 (1987), 161-186.

, Priority rules and other asymmetric rationing methods, Econometrica 68 (2000).

, Axiomatic cost and surplus-sharing, Handbook of Social Choice and Welfare (Sen Arrow and Suzumura, eds.), 2002.

H. Moulin and S. Shenker, Serial cost sharing, Econometrica 60 (1992), 1009-1037.

H Moulin and S. Shenker, Average cost pricing versus serial cost sharing; an axiomatic comparison, Journal of Economic Theory 64 (1994), 178-201.

W. Sharkey, The theory of natural monopoly, Cambridge University Press, Cambridge, U.K., 1982.

M. Shubik, Incentives, decentralized control, the assignment of joint cost, and internal pricing, Management Science 8 (1962), 325-343.

A.I. Sobolev, Characterization of the principle of optimality for cooperative games through functional equations, Mathematical Methods in the Social Sciences (Vorobyev, ed.), Vipusk 6, Vilnius, USSR, 1975.

Y. Sprumont, Ordinal cost sharing, Journal of Economic Theory 81 (1998), 126-162.

P. Sudhölter, Axiomatizations of game theoretical solutions for one-output cost sharing problems, Games and Economic Behavior 24 (1998), 42-71.

W. Thomson, Consistent allocation rules, Mimeo, Economics Department, University of Rochester, Rochester, U.S.A., 1996.

, On the axiomatic method and its applications to game theory and resource allocation, forthcoming in Social Choice and Welfare, 1999. 
S. H. Tijs and M. Koster, General aggregation of demand and cost sharing methods, Annals of Operations Research 84 (1998), 137-164.

R. Weber, Probabilistic values for games, The Shapley Value (Roth, ed.), Cambridge University Press, Cambridge, U.K., 1988.

H. P. Young, Cost allocation: Methods, principles, applications, North-Holland, Amsterdam, The Netherlands, 1985.

On dividing an amount according to individual claims or liabilities, Mathematics of Operations Research 12 (1987), 398-414.

Cost allocation, Handbook of Game Theory II (R.J. Aumann and S. Hart, eds.), Elsevier, Amsterdam, The Netherlands, 1994, pp. 1193-1235.

\section{A Appendix}

Lemma A.1 Let $R=(q, f) \in \mathcal{R}^{S}$ and take $y \in \mathcal{C}^{\mathrm{P}}(R)$. Then for each $i \in S$ there is $a \in[0, q(S \backslash\{i\})]$ such that $y_{i}=f_{R}^{\mathrm{P}}\left(a+q_{i}\right)-f_{R}^{\mathrm{P}}(a)$.

Proof. Let $i \in S$ and define $g:[0, \infty) \rightarrow \mathbb{R}$ by $g(t)=f_{R}^{\mathrm{P}}\left(t+q_{i}\right)-f_{R}^{\mathrm{P}}(t)$. Then $g(0)=f_{R}^{\mathrm{P}}\left(q_{i}\right) \leq y_{i}$ and $g(q(S \backslash\{i\}))=f_{R}^{\mathrm{P}}(q(S))-f_{R}^{\mathrm{P}}(q(S \backslash\{i\})) \geq y_{i}$. The latter inequality follows from the fact that $y \in \mathcal{C}^{\mathrm{P}}(q, f)$ since $f_{R}^{\mathrm{P}}(q(S))=y(S)$ and $f_{R}^{\mathrm{P}}(q(S \backslash\{i\})) \leq y(S \backslash\{i\})$. Recall that $f_{R}^{\mathrm{P}}$ is convex and thus continuous. Then by continuity of $g$ there exists $a$ such that $g(a)=y_{i}$.

Lemma A.2 Let $R=(q, f) \in \mathcal{R}^{S}$. For each $x \in[0, q(S)]$ there is $T_{x} \in \mathcal{B}([0, q(S)])$ such that $f_{R}^{\mathrm{P}}(x)=\int_{T_{x}} f^{\prime}(t) d t$ and $\lambda\left(T_{x}\right)=x$. The sets can be taken such that $x \leq y \Longrightarrow T_{x} \subseteq T_{y}$.

Proof. Take $x \in[0, q(S)]$. For $z \in \mathbb{R}_{+}$we define $D_{z}:=\left\{t \in[0, q(S)] \mid f^{\prime}(t) \geq z\right\}$. Then let $z(x):=$ $\sup \left\{z \in \mathbb{R}_{+} \mid \lambda\left(D_{z}\right) \geq x\right\}$. We distinguish two cases, $\lambda\left(D_{z}\right)=x$ and $\lambda\left(D_{z(x)}\right)>x$. We will show that the choice of $T_{x}:=D_{z(x)}$ serves our goal. To see this, just take an arbitrary $T \in \mathcal{B}([0, q(S)])$ with $\lambda(T)=x, T \neq T_{x}$. Then in particular for $t \in T \backslash T_{x}$ it holds that $f^{\prime}(t)<z(x)$ and therefore

$$
\int_{T \backslash T_{x}} f^{\prime}(t) \mathrm{d} t \leq z(x) \cdot \lambda\left(T_{x} \backslash T\right) \leq \int_{T_{x} \backslash T} f^{\prime}(t) \mathrm{d} t .
$$

As a consequence

$$
\begin{aligned}
\int_{T_{x} \backslash T} f^{\prime}(t) \mathrm{d} t & =\int_{T \cap T_{x}} f^{\prime}(t) \mathrm{d} t+\int_{T \backslash T_{x}} f^{\prime}(t) \mathrm{d} t \\
& \leq \int_{T \cap T_{x}} f^{\prime}(t) \mathrm{d} t+\int_{T_{x} \backslash T} f^{\prime}(t) \mathrm{d} t \\
& =\int_{T_{x}} f^{\prime}(t) \mathrm{d} t .
\end{aligned}
$$


So $f_{R}^{\mathrm{P}}(x)=\sup \left\{\int_{T} f^{\prime}(t) \mathrm{d} t \mid \lambda(T)=x\right\}=\int_{T_{x}} f^{\prime}(t) \mathrm{d} t$. Now for the second case assume that $\lambda\left(D_{z(x)}\right)>$ $x$. This means that

$$
\lambda\left(\left\{t \in[0, q(S)] \mid f^{\prime}(t)=z(x)\right\}\right)>\lambda\left(D_{z(x)}\right)-x .
$$

Determine $t^{\prime} \in[0, q(S)]$ with

$$
\lambda\left(\left[0, t^{\prime}\right] \cap\left\{t \in[0, q(S)] \mid f^{\prime}(t)=z(x)\right\}\right)=\lambda\left(D_{z(x)}\right)-x .
$$

Now take $T_{x}:=D_{z(x)} \backslash\left(\left[0, t^{\prime}\right] \cap\left\{t \in[0, q(S)] \mid f^{\prime}(t)=z(x)\right\}\right)$. Then $\lambda\left(T_{x}\right)=x$ and the rest is proved analogously to the first case. Besides, it should be clear from the presented construction that $T_{x} \subseteq T_{y}$ whenever $x \leq y$.

\section{Lemma A.3}

Let $f:[0, y] \rightarrow \mathbb{R}$ be increasing and convex such that $f(0)=0$. Then for any $\alpha \in[0,1]$ there is an interval $I=[t, t+\alpha y] \subseteq[0, y]$ such that

$$
\alpha f(y)=f(t+\alpha y)-f(t) .
$$

Proof. Define $g:[0,(1-\alpha) y] \rightarrow \mathbb{R}_{+}$by $g(t)=f(t+\alpha y)-f(t)$. Convexity of $f$ implies

$$
g(0)=f(\alpha y) \leq \alpha f(y)=f(y)-(1-\alpha y) f(y) \leq f(y)-f((1-\alpha) y)=g((1-\alpha) y) .
$$

Hence by continuity of $g$ there is $t \in[0,(1-\alpha) y]$ such that $g(t)=\alpha f(y)$.

Lemma A.4 For any $\mu \in \mathcal{S}(\mathrm{ADD}, \mathrm{POS}, \mathrm{CR}), Q(\mu, R, i) \neq \varnothing$ for all $R \in \mathcal{R}$.

Proof. Take $R=(q, f) \in \mathcal{R}^{S}, i \in S$. By Theorem 4.7 and Lemma A.1 there is an interval $T=\left[a, a+q_{i}\right]$ such that $\int_{T} f^{\prime}(t) \mathrm{d} t=f_{R}^{\mathrm{P}}\left(a+q_{i}\right)-f_{R}^{\mathrm{P}}(a)$. Moreover we may choose sets $U, V \in \mathcal{B}([0, q(S)]), U \subseteq V$ such that $\int_{U} f^{\prime}(t) \mathrm{d} t=f_{R}^{\mathrm{P}}(a)$ and $\int_{V} f^{\prime}(t) \mathrm{d} t=f_{R}^{\mathrm{P}}\left(a+q_{i}\right)$. Then $V \backslash U \in Q(\mu, R, i)$.

\section{Proof of Proposition 4.2}

Let $\mu \in \mathcal{M}^{+}(\mathrm{ADD})$. If there is an extension of $\mu$ to $\mathcal{R}$ then it must be $\bar{\mu} \in \mathcal{M}$ defined by $\bar{\mu}(q, f)=$ $\mu\left(q, f^{+}\right)-\mu\left(q, f^{-}\right)$for all $(q, f) \in \mathcal{R}$. We will show that $\bar{\mu} \in \mathcal{M}($ ADD $)$. Take any $f_{1}, f_{2} \in \mathcal{F}_{+}$. Then we have $f_{1}^{+}+f_{2}^{+}-\left(f_{1}+f_{2}\right)^{+} \in \mathcal{F}_{+}$and $\left(f_{1}+f_{2}\right)^{-}-f_{1}^{-}-f_{2}^{-} \in \mathcal{F}_{+}$and

$$
\begin{aligned}
& \bar{\mu}\left(q, f_{1}\right)+\bar{\mu}\left(q, f_{2}\right)-\bar{\mu}\left(q, f_{1}+f_{2}\right)= \\
& \mu\left(q, f_{1}^{+}\right)-\mu\left(q, f_{1}^{-}\right)+\mu\left(q, f_{2}^{+}\right)-\mu\left(q, f_{2}^{-}\right)-\mu\left(q,\left(f_{1}+f_{2}\right)^{+}\right)+\mu\left(q,\left(f_{1}+f_{2}\right)^{-}\right)= \\
& \mu\left(q, f_{1}^{+}+f_{2}^{+}\right)-\mu\left(q,\left(f_{1}+f_{2}\right)^{+}\right)+\mu\left(q,\left(f_{1}+f_{2}\right)^{-}\right)-\mu\left(q, f_{1}^{-}+f_{2}^{-}\right)= \\
& \mu\left(q, f_{1}^{+}+f_{2}^{+}-\left(f_{1}+f_{2}\right)^{+}\right)+\mu\left(q,\left(f_{1}+f_{2}\right)^{-}-f_{1}^{-}-f_{2}^{-}\right)= \\
& \mu\left(q,\left(f_{1}^{+}+f_{2}^{+}-\left(f_{1}+f_{2}\right)^{+}\right)+\left(\left(f_{1}+f_{2}\right)^{-}-f_{1}^{-}-f_{2}^{-}\right)\right)= \\
& \mu\left(q, f_{1}+f_{2}-\left(f_{1}+f_{2}\right)\right)=0 .
\end{aligned}
$$

This proves ADD. 


\section{Proof of Theorem 4.3}

Take $\mu \in \mathcal{M}$ (POS, ADD, CR). Then in particular $\mu \in \mathcal{M}^{+}$(POS, ADD, CR). By Theorem 4.1 there is a rationing family $\pi$ for $\mu$. Then by Proposition 4.2 there is a unique extension $\bar{\mu}$ of $\mu$ to $\mathcal{M}$ (ADD) and

$$
\begin{aligned}
\bar{\mu}(q, f) & =\mu\left(q, f^{+}\right)-\mu\left(q, f^{-}\right) \\
& =\int_{0}^{q(S)}\left(f^{+}\right)^{\prime}(t) \mathrm{d} r(q, t)-\int_{0}^{q(S)}\left(f^{-}\right)^{\prime}(t) \mathrm{d} r(q, t) \\
& =\int_{0}^{q(S)}\left(f^{+}-f^{-}\right)^{\prime}(t) \mathrm{d} r(q, t)=\int_{0}^{q(S)} f^{\prime}(t) \mathrm{d} r(q, t) .
\end{aligned}
$$

Moreover $\mu=\bar{\mu}$ and we are there. The other way around is easy.

\section{Proof of Theorem 4.7.}

The proof of the inclusion "卫" is easy and essentially done in Tijs and Koster (1998). We will now prove " $\subseteq$ ". Basically the argument is to choose a partition of the interval $[0, q(S)]$ such that each member in this partition is associated with a particular agent such that the corresponding marginal profits equal the share according to $y \in \mathcal{C}^{\mathrm{P}}(q, f)$. Then this partition is used to define a rationing method $r(q, \cdot)$. Then any set of rationing methods for other input profiles completes the definition of the necessary rationing family $\pi$. At this point we may assume without loss of generality that $S=N$ and that we made a choice for the rationing methods $r\left(q^{\prime}, \cdot\right)$ for all $q^{\prime} \neq q$. Then our objective is to find $r(q, \cdot)$ such that $\mu^{\pi}(R)=y$. Let $T_{1}=\left[a_{1}, a_{1}+q_{1}\right]$ be an interval as in Lemma A.1 for agent 1 and let $f_{1}=f_{R}^{\mathrm{p}}$. Define a new profits sharing problem $R_{2}=\left(q_{2}, f_{2}\right)$ by $q_{2}:=q_{N \backslash\{1\}}$ and

$$
f_{2}(y)= \begin{cases}f_{1}(y) & \text { if } y \leq a_{1} \\ f_{1}\left(a_{1}\right)+f_{1}\left(y+q_{1}\right)-f\left(a_{1}+q_{1}\right) & \text { if } y>a_{1} .\end{cases}
$$

Notice that $f_{2}$ is convex and that for this reason $f_{R_{2}}^{\mathrm{P}}=f_{2}$. Application of Lemma A.1 to $R_{2}$ and agent 2 gives us a set $T_{2}=\left[a_{2}, a_{2}+q_{2}\right]$ such that $f_{2}\left(a_{2}+q_{2}\right)-f_{2}\left(a_{2}\right)=y_{2}$. Then we proceed as follows. Having defined $R_{2}, R_{3}, \ldots, R_{i}$ and intervals $T_{1}, T_{2}, \ldots, T_{i}, T_{k}=\left[a_{k}, a_{k}+q_{k}\right] \subseteq\left[0, \sum_{j \geq k} q_{j}\right]$, we define $R_{i+1}$ by $\left(q_{N \backslash\{1,2, \ldots, i\}}, f_{i+1}\right)$ where $f_{i+1} \in \mathcal{F}$ is given by

$$
f_{i+1}(y)= \begin{cases}f_{i}(y) & \text { if } 0 \leq y \leq a_{i} \\ f_{i}\left(a_{i}\right)+f_{i}\left(y+q_{i}\right)-f_{i}\left(a_{i}+q_{i}\right) & \text { if } y>a_{i}\end{cases}
$$

Notice that $f_{i+1}$ is convex on $\left[0, \sum_{j \geq i+1} q_{j}\right]$ such that $f_{R_{i+1}}^{\mathrm{P}}=f_{i+1}$. Then by application of Lemma A.1 there is a $a_{i+1} \in\left[0, \sum_{j \geq i+2} q_{j}\right]$ with the property that $f_{i+1}\left(a_{i+1}+q_{i+1}\right)-f_{i+1}\left(a_{i+1}\right)=y_{i+1}$. Then define $T_{i+1}=\left[a_{i+1}, a_{i+1}+q_{i+1}\right]$. Define for each $i \in N$ a function $g_{i}:[0, \infty) \rightarrow \mathbb{R}_{+}$by

$$
g_{i}(y)= \begin{cases}y & \text { if } y \leq a_{i} \\ y+q_{i} & \text { if } y>a_{i}\end{cases}
$$

In addition define $U_{1}:=T_{1}$ and for $i \in N \backslash\{1\}$ let $U_{i} \subseteq[0, q(N)]$ be defined by $U_{i}:=\left(g_{2} \circ g_{3} \circ \ldots \circ g_{i}\right)\left(T_{i}\right)$. Then these sets $U_{1}, U_{2}, \ldots, U_{n}$ have the following properties

$$
\begin{cases}\lambda\left(U_{i} \cap U_{j}\right)=0 & \text { if } i \neq j, \text { and } \\ \lambda\left(U_{i}\right)=\lambda\left(T_{i}\right)=q_{i} & \text { for all } i \in N\end{cases}
$$


Assume without loss of generality that $\left\{U_{i}\right\}_{i \in N}$ constitutes a partition of $[0, q(S)]$. Now define the rationing method $r(q, \cdot)$ by

$$
r_{k}(q, t)=\int_{0}^{t} \mathbb{I}_{U_{k}}(s) \mathrm{d} s \text { for all } t \in[0, q(S)], k \in N
$$

Then

$$
\begin{aligned}
y_{k} & =\int_{T_{k}} f_{k}^{\prime}(t) \mathrm{d} t=\int_{g_{k}\left(T_{k}\right)} f_{k-1}^{\prime}(t) \mathrm{d} t=\ldots=\int_{\left(g_{2} \circ g_{3} \circ \ldots \circ g_{k}\right)\left(T_{k}\right)} f_{1}^{\prime}(t) \mathrm{d} t \\
& =\int_{U_{k}}\left(f_{R}^{\mathrm{P}}\right)^{\prime}(t) \mathrm{d} t=\int_{0}^{q(S)} \mathbb{I}_{U_{k}}(t)\left(f_{R}^{\mathrm{P}}\right)^{\prime}(t) \mathrm{d} t=\int_{0}^{q(S)}\left(f_{R}^{\mathrm{P}}\right)^{\prime}(t) \mathrm{d} r_{k}(q, t) .
\end{aligned}
$$

\section{Proof of Theorem 5.5}

Step 1: First I will show that any incremental mechanism $\mu \in \mathcal{M}(\mathrm{POS}, \mathrm{ADD}, \mathrm{CR})$ defined by (10) is consistent if and only if for all $S \subseteq N, q \in \mathbb{R}_{+}^{S}$ and $j \in S$ it holds that for all $f \in \mathcal{F}$

$$
\mu\left(q_{S \backslash\{j\}}, f\right)=\sum_{\ell=1}^{k} \tilde{\alpha}_{\ell}\left\{f\left(\tilde{x}_{\ell}\right)-f\left(\tilde{x}_{\ell-1}\right)\right\},
$$

where $\tilde{x} \in \mathbb{R}^{k+1}$ and $\tilde{\alpha}_{1}, \tilde{\alpha}_{2}, \ldots, \tilde{\alpha}_{k} \in \Delta(S \backslash\{j\})$ are given for each $\ell=1,2, \ldots, k$ by

$$
\begin{aligned}
& \tilde{x}_{\ell}=\sum_{1 \leq p \leq \ell}\left(1-\alpha_{p j}\right)\left(x_{p}-x_{p-1}\right), \\
& \tilde{\alpha}_{\ell}= \begin{cases}\frac{\alpha_{\ell}}{\alpha_{\ell}(S \backslash\{j\})} & \text { if } \alpha_{\ell}(S \backslash\{j\})>0, \\
0 & \text { else. }\end{cases}
\end{aligned}
$$

Let $R=(q, f) \in \mathcal{R}^{S}, j \in S$ and $\alpha, x$ be as in (10) and $\tilde{\alpha}, \tilde{x}$ as in (15) and (16), respectively. We will show that there is a set $U \in \mathcal{Q}(\mu, R, j)$ such that $\mu_{S \backslash\{j\}}(q, f)=\mu\left(q_{S \backslash\{j\}}, f_{U}\right)$. Define for each $\ell \in\{1,2, \ldots, k\}$ the convex function $f_{\ell}:\left[x_{\ell-1}, x_{\ell}\right] \rightarrow \mathbb{R}$ by

$$
f_{\ell}(y)=\inf \left\{\int_{T} f^{\prime}(t) \mathrm{d} t \mid T \in \mathcal{B}\left(\left[x_{\ell-1}, x_{\ell}\right]\right), \lambda(T)=y\right\} .
$$

According to Lemma A.3 (in the Appendix) there is for each $\ell$ an interval $I_{\ell}=\left[t_{\ell}, t_{\ell}+\alpha_{\ell j}\left(x_{\ell}-x_{\ell-1}\right)\right] \subset$ $\left[x_{\ell-1}, x_{\ell}\right]$ such that $f_{\ell}\left(t_{\ell}+\alpha_{\ell j}\left(x_{\ell}-x_{\ell-1}\right)\right)-f_{\ell}\left(t_{\ell}\right)=\alpha_{\ell j} f_{\ell}\left(x_{\ell}-x_{\ell-1}\right)$. As in Lemma A.2 each interval $I_{\ell}$ corresponds to a set $U_{\ell} \in \mathcal{B}\left(\left[x_{\ell-1}, x_{\ell}\right]\right)$ such that

$$
\int_{U_{\ell}} f^{\prime}(t) \mathrm{d} t=\alpha_{\ell j} f_{\ell}\left(\left(x_{\ell}-x_{\ell-1}\right)=\alpha_{\ell j}\left(f\left(x_{\ell}\right)-f\left(x_{\ell-1}\right)\right) .\right.
$$

Then in particular $U=\cup_{\ell} U_{\ell} \in \mathcal{Q}(\mu, R, j)$. By construction we have

$$
f_{U}\left(\tilde{x}_{\ell}\right)-f_{U}\left(\tilde{x}_{\ell-1}\right)=\left(1-\alpha_{\ell j}\right)\left(f\left(x_{\ell}\right)-f\left(x_{\ell-1}\right)\right) .
$$


Hence for all $i \in S \backslash\{j\}$

$$
\begin{aligned}
\mu_{i}\left(q_{S \backslash\{j\}}, f_{U}\right) & =\sum_{\ell=1}^{k} \tilde{\alpha}_{\ell i}\left(f_{U}\left(\tilde{x}_{\ell}\right)-f_{U}\left(\tilde{x}_{\ell-1}\right)\right)=\sum_{\ell ; \alpha_{\ell j} \neq 1} \frac{\alpha_{\ell i}}{1-\alpha_{\ell j}} \cdot\left(1-\alpha_{\ell j}\right)\left(f\left(x_{\ell}\right)-f\left(x_{\ell-1}\right)\right) \\
& =\sum_{\ell ; \alpha_{\ell j} \neq 1} \alpha_{\ell i}\left(f\left(x_{\ell}\right)-f\left(x_{\ell-1}\right)\right)=\sum_{\ell=1}^{k} \alpha_{\ell i}\left(f\left(x_{\ell}\right)-f\left(x_{\ell-1}\right)\right)=\mu_{i}(q, f) .
\end{aligned}
$$

Notice that the fourth equality is due to the fact that $\alpha_{\ell j}$ implies $\alpha_{\ell i}=0$.

Now the second part of the statement. Suppose that $\mu \in \mathcal{M}^{I}(\mathrm{POS}, \mathrm{ADD}, \mathrm{CR}, \mathrm{CO})$ and that for $q \in \mathbb{R}_{+}^{S}$ there are $x \in \mathbb{R}^{k+1}$ and $\tilde{\alpha}_{1}, \tilde{\alpha}_{2}, \ldots, \tilde{\alpha}_{k} \in \Delta(S)$ as in (10) and (11). We will show that the fact that $\mu$ is consistent implies the equalities (15) and (16). Lemma 5.11 below shows that the vectors $x \in \mathbb{R}_{+}^{k+1}$ and $\alpha_{1}, \alpha_{2}, \ldots, \alpha_{k}$ can be used together with (15) and (16) to define a mechanism $\mu^{\pi} \in \mathcal{M}^{I}$ (POS, ADD, CR, CO) such that $\mu(q, f)=\mu^{\pi}(q, f)$ for all $f \in \mathcal{F}$. We will show that $\mu\left(q_{S \backslash\{j\}}, f\right)=\mu^{\pi}\left(q_{S \backslash\{j\}}, f\right)$ for all $f \in \mathcal{F}$. For $t \in \mathbb{R}_{+}$we define $R_{t}=\left(q, \Lambda_{t}\right)$ where $\Lambda_{t}: \mathbb{R}_{+} \rightarrow \mathbb{R}_{+}$is the slant function defined by $\Lambda_{t}(y)=\max \{y-t, 0\}$ for all $y \in \mathbb{R}_{+}$. We will show that $\mu\left(q_{S \backslash\{j\}}, \Lambda_{t}\right)=\mu^{\pi}\left(q_{S \backslash\{j\}}, \Lambda_{t}\right)$ for all $t \geq 0$. Obviously this holds whenever $t>q(S \backslash\{j\})$ as $\Lambda_{t}(y)=0$ for all $y \in[0, q(S \backslash\{j\})]$. Consider the map $h:[0, q(S \backslash\{j\})] \rightarrow \mathbb{R}_{+}$defined by $h(t)=t-\mu_{j}\left(q, \Lambda_{t}\right)$. Then $h$ is non-decreasing and continuous and, moreover, $h([0, q(S)])=\left[0, q_{S \backslash\{j\}}\right]$. This means that for each $t \in[0, q(S \backslash\{j\})]$ and $t^{*} \in h^{-1}(t)$ that for each $U \in \mathcal{Q}\left(\mu, R_{t}, j\right)$ and $V \in \mathcal{Q}\left(\mu^{\pi}, R_{t}, j\right)$ where $R_{t}=\left(q, \Lambda_{t}\right)$ it holds

$$
\left(\Lambda_{t^{*}}\right)_{U}=\left(\Lambda_{t^{*}}\right)_{V}=\Lambda_{t}
$$

Then

$$
\begin{aligned}
\mu^{\pi}\left(q_{S \backslash\{j\}}, \Lambda_{t}\right) & =\mu^{\pi}\left(q_{S \backslash\{j\}},\left(\Lambda_{t^{*}}\right)_{V}\right)=\mu_{S \backslash\{j\}}^{\pi}\left(q, \Lambda_{t^{*}}\right)= \\
& =\mu_{S \backslash\{j\}}\left(q, \Lambda_{t^{*}}\right)=\mu\left(q_{S \backslash\{j\}^{\prime}}\left(\Lambda_{t^{*}}\right)_{U}\right)= \\
& =\mu\left(q_{S \backslash\{j\}},\left(\Lambda_{t^{*}}\right)_{U}\right)=\mu\left(q_{S \backslash\{j\}}, \Lambda_{t}\right) .
\end{aligned}
$$

Moulin and Shenker (1994) show that two additive mechanisms coincide whenever they yield the same share vectors for slant functions. The same reasoning can be adopted here in order to conclude that $\mu\left(q_{S \backslash\{j\}}, f\right)=\mu^{\pi}\left(q_{S \backslash\{j\}}, f\right)$ for all $f \in \mathcal{F}$. Now, finally, observe that two incremental mechanisms only determine the same share vectors if they allow for the same formulation in terms of $x$ and $\alpha$ 's as in (10).

\section{Step 2:}

Suppose $r$ belongs to a family $\pi$ of consistent and piecewise linear rationing methods, and that for $q \in \mathbb{R}_{+}^{S}$ there are $x_{0}, x_{1}, \ldots, x_{k}$ with $0=x_{0}<\ldots<x_{k}=q(S)$ and $\alpha_{1}, \ldots, \alpha_{k} \in \Delta(S)$ such that for any $\ell \in\{1,2, \ldots, k\}$ and $t \in\left[x_{\ell-1}, x_{\ell}\right]$

$$
r(q, t)=\sum_{h=1}^{\ell-1} \alpha_{h}\left(x_{h}-x_{h-1}\right)+\alpha_{\ell}\left(t-x_{\ell-1}\right)
$$


Now I will prove that $r$ is consistent if and only if for all $t \in\left[\tilde{x}_{\ell-1}, \tilde{x}_{\ell}\right]$

$$
r\left(q_{S \backslash\{j\}}, t\right)=\sum_{h=1}^{\ell-1} \tilde{\alpha}_{h}\left(\tilde{x}_{h}-\tilde{x}_{h-1}\right)+\tilde{\alpha}_{\ell}\left(t-\tilde{x}_{\ell-1}\right),
$$

where $\tilde{x}_{\ell}, \tilde{\alpha}_{\ell}$ are defined by the equalities (15) and (16). First notice that by piecewise linearity the mappings $t \mapsto r(q, t)$ and $y \mapsto r\left(q_{S \backslash\{j\}}, y\right)$ are both differentiable almost everywhere. Hence, by consistency, it holds for $i \in S \backslash\{j\}$ and almost all $t \in\left[x_{\ell-1}, x_{\ell}\right]$ that

$$
\begin{aligned}
\alpha_{\ell i} & =\frac{\partial}{\partial t} r_{i}(q, t)=\frac{\partial}{\partial t} r_{i}\left(q_{S \backslash\{i\}}, t-r_{j}(q, t)\right) \\
& =\left.\frac{\partial}{\partial y} r_{i}\left(q_{S \backslash\{j\}}, y\right)\right|_{y=t-r_{j}(q, t)} \cdot \frac{\partial}{\partial t}\left(t-r_{j}(q, t)\right) \\
& =\left.\frac{\partial}{\partial y} r_{i}\left(q_{S \backslash\{j\}}, y\right)\right|_{y=t-r_{j}(q, t)} \cdot\left(1-\alpha_{\ell j}\right) \\
& =\left.\frac{\partial}{\partial y} r_{i}\left(q_{S \backslash\{i\}}, y\right)\right|_{y=t-r_{j}(q, t)} \cdot \alpha_{\ell}(S \backslash\{j\}) .
\end{aligned}
$$

The constant $\alpha_{\ell}(S \backslash\{j\})=0$ if and only if $x_{\ell-1}-r_{j}\left(q, x_{\ell-1}\right)=x_{\ell-1}-r_{j}\left(q, x_{\ell-1}\right)$, hence we may conclude that for any $y \in\left(x_{\ell-1}-r_{j}\left(q, x_{\ell-1}\right), x_{\ell-1}-r_{j}\left(q, x_{\ell-1}\right)\right)$ the derivative of the mapping $y \mapsto$ $r\left(q_{S \backslash\{j\}}, y\right)$ is constant. Now define $\bar{x}_{0}, \bar{x}_{1}, \ldots, \bar{x}_{k}$ and $\bar{\alpha}_{1}, \bar{\alpha}_{2}, \ldots, \bar{\alpha}_{k}$ by

$$
\begin{aligned}
& \bar{x}_{\ell}=x_{\ell}-r_{j}\left(q, x_{\ell}\right) \\
& \bar{\alpha}_{\ell}= \begin{cases}\alpha_{\ell} \cdot \alpha_{\ell}(S \backslash\{j\})^{-1} & \text { if } \alpha(S \backslash\{j\})>0, \\
0 & \text { else }\end{cases}
\end{aligned}
$$

Then it holds that for all $t \in\left[\bar{x}_{\ell-1}, \bar{x}_{\ell}\right]$

$$
r\left(q_{S \backslash\{j\}}, t\right)=\sum_{h=1}^{\ell-1} \bar{\alpha}_{h}\left(\bar{x}_{h}-\bar{x}_{h-1}\right)+\bar{\alpha}_{\ell}\left(t-\bar{x}_{\ell-1}\right) .
$$

Notice that the above definitions of $\bar{x}_{\ell}$ and $\bar{\alpha}_{\ell}$ match that of $\tilde{x}_{\ell}$ and $\tilde{\alpha}_{\ell}$ in equations (15) and (16), respectively, since

$$
\begin{aligned}
x_{\ell}-r_{j}(q, t) & =x_{\ell}-\sum_{h=1}^{\ell} \alpha_{h j}\left(x_{h}-x_{h-1}\right) \\
& =\sum_{h=1}^{\ell}\left(x_{h}-x_{h-1}\right)-\sum_{h=1}^{\ell} \alpha_{h j}\left(x_{h}-x_{h-1}\right) \\
& =\sum_{h=1}^{\ell}\left(1-\alpha_{h j}\right)\left(x_{h}-x_{h-1}\right) .
\end{aligned}
$$


Then Step 1 together with Step 2 complete the proof of the theorem.

\section{Proof of Theorem 5.7}

Suppose $\mu$ is an interval consistent incremental mechanism. Then in particular $\mu$ is consistent. Consider $q \in \mathcal{R}_{++}^{S}$. Then there is $k \in \mathbb{N}$ and for each $\ell \in\{1,2, \ldots, k\}$ a real number $x_{\ell} \in[0, q(S)]$ and vector $\alpha_{\ell} \in \Delta(S)$ as in (definition) such that

$$
\mu(q, f)=\sum_{\ell=1}^{k} \alpha_{\ell}\left\{f\left(x_{\ell}\right)-f\left(x_{\ell-1}\right)\right\}, \text { for all } f \in \mathcal{F} .
$$

Consider functions $f_{n}: \mathbb{R}_{+} \rightarrow \mathbb{R}$ by $f_{n}(x)=e^{n x}-1$ for all $x \geq 0, n \in \mathbb{N}$ and define $R_{n}=\left(q, f_{n}\right)$. Then for each $n$ such $f_{n}$ is strictly convex. In particular this means that for each $j \in N$ there is precisely one interval $I(n, j)=\left[t_{n}^{j}, t_{n}^{j}+q_{j}\right] \in \mathcal{Q}\left(\mu, R_{n}, j\right)$ for each $n \in \mathbb{N}$. Define $f_{n}^{j}=\left(f_{n}\right)_{I(n, j)}$ for each $n \in \mathbb{N}$. Distinguish the following cases: (i) there is only one agent $j$ with positive $\alpha_{k j}$, or $\alpha_{k j}=1$, and (ii) there are at least two agents $i$ and $j$ with $\alpha_{k i}, \alpha_{k j}>0$.

Case (i): Suppose that $x_{k}-x_{k-1}<q_{j}$. Then $t_{n}^{j}<x_{k-1}$ for all $n \in \mathbb{N}$ and for large $n$ it holds

$$
\begin{aligned}
\mu_{j}\left(q, f_{n}\right) & \geq \alpha_{k j}\left\{f_{n}\left(x_{k}\right)-f_{n}\left(x_{k-1}\right)\right\} \\
& =f_{n}\left(x_{k}\right)-f_{n}\left(x_{k-1}\right) \\
& >f_{n}\left(t_{n}^{j}+q_{j}\right)-f_{n}\left(t_{n}^{j}\right) .
\end{aligned}
$$

But this means that $I(n, j) \notin \mathcal{Q}\left(\mu, R_{n}, j\right)$ for large $n$, contradiction. Hence it must be that $x_{k}-x_{k-1}=q_{j}$. Then $\alpha_{\ell j}=0$ for all $\ell \neq k$.

Case (ii): Suppose that there is a subsequence $\left\{t_{h(n)}^{j}\right\}_{n \in \mathbb{N}}$ of $\left\{t_{n}^{j}\right\}_{n \in \mathbb{N}}$ such that $t_{h(n)}^{j} \leq \tilde{x}_{k-1}$ for all $n \in \mathbb{N}$. Since $\mu$ is interval consistent we have for each $n \in \mathbb{N}$ that $\mu_{S \backslash\{j\}}\left(q, f_{n}\right)=\mu\left(q_{S \backslash\{j\}}, f_{n}^{j}\right)$. By Step $l$ in the proof of Theorem 5.5 it must hold for all $n \in \mathbb{N}, i \in S \backslash\{j\}$

$$
\sum_{\ell=1}^{k} \alpha_{\ell i}\left\{f_{n}\left(x_{\ell}\right)-f_{n}\left(x_{\ell-1}\right)\right\}-\tilde{\alpha}_{\ell i}\left\{f_{n}^{j}\left(\tilde{x}_{\ell}\right)-f_{n}^{j}\left(\tilde{x}_{\ell-1}\right)\right\}=0,
$$

By distinguishing the powers in this sum we must have

$$
\lim _{n \rightarrow \infty} \alpha_{k i}\left\{f_{n}\left(x_{k}\right)-f_{n}\left(x_{k-1}\right)\right\}-\tilde{\alpha}_{k i}\left\{f_{n}^{j}\left(\tilde{x}_{k}\right)-f_{n}^{j}\left(\tilde{x}_{k-1}\right)\right\}=0 .
$$

For all $n$ we have

$$
f_{h(n)}^{j}\left(\tilde{x}_{k}\right)-f_{h(n)}^{j}\left(\tilde{x}_{k-1}\right)=f_{h(n)}\left(\tilde{x}_{k}+q_{j}\right)-f_{h(n)}\left(\tilde{x}_{k-1}\right)-\mu_{j}\left(q, f_{h(n)}\right) .
$$

Then use the expression for $\mu_{j}\left(q, f_{h(n)}\right)$ to see that (22) is equivalent with

$$
\begin{aligned}
& \lim _{n \rightarrow \infty} \alpha_{k i}\left\{f_{h(n)}\left(x_{k}\right)-f_{h(n)}\left(x_{k-1}\right)\right\}+ \\
& -\tilde{\alpha}_{k i}\left\{f_{h(n)}\left(x_{k}\right)-f_{h(n)}\left(x_{k-1}-q_{j}+\alpha_{k j}\left(x_{k}-x_{k-1}\right)\right)-\alpha_{k j}\left(f_{h(n)}\left(x_{k}\right)-f_{h(n)}\left(x_{k-1}\right)\right)\right\}=0 .
\end{aligned}
$$


Since $\alpha_{k i}-\tilde{\alpha}_{k i}\left(1-\alpha_{j \ell}\right)=0$, the terms with the highest argument $x_{k}$ vanish, so we get

$$
\lim _{n \rightarrow \infty}-\alpha_{k i} f_{h(n)}\left(x_{k-1}\right)-\tilde{\alpha}_{k i}\left\{-f_{h(n)}\left(x_{k-1}-q_{j}+\alpha_{k j}\left(x_{k}-x_{k-1}\right)\right)+\alpha_{k j} f_{h(n)}\left(x_{k-1}\right)\right\}=0 .
$$

Since $\alpha_{k i}>0$ it must hold that $\alpha_{k j}\left(x_{k}-x_{k-1}\right)=q_{j}$. By interchanging the role of $i$ and $j$ we see that also $\alpha_{k i}\left(x_{k}-x_{k-1}\right)=q_{i}$. In particular for any agent $p$ with $\alpha_{k p}>0$ it holds that $\alpha_{k p}\left(x_{k}-x_{k-1}\right)=q_{p}$ and therefore

$$
x_{k}-x_{k-1}=\sum_{j \in S ; \alpha_{k j}>0} \alpha_{k j}\left(x_{k}-x_{k-1}\right)=\sum_{j \in S ; \alpha_{k j}>0} q_{j},
$$

hence for each $i$ with $\alpha_{k i}>0$ it holds that

$$
\alpha_{k i}=\frac{q_{i}}{\sum_{j \in S ; \alpha_{k j}>0} q_{j}} \text { and } \alpha_{\ell i}=0 \text { for all } \ell \neq k
$$

\section{Proof of Theorem 5.10}

Part (i): Take $y \in \mathcal{C}^{\mathrm{P}}(q, f)$ for $(q, f) \in \mathcal{R}^{S}, S \subseteq N$. Let $i \in S, Q \subset S \backslash\{i\}$. According to Lemma A.1 there is an interval $\left[a, a+q_{i}\right] \subseteq[0, q(S)]$ such that $y_{i}=f_{R}^{\mathrm{P}}\left(a+q_{i}\right)-f_{R}^{\mathrm{P}}(a)$. Consider a family of measurable sets $\left\{T_{z}\right\}_{z \in[0, q(S)]}$ as in Lemma A.2. Consider $T=T_{a+q_{i}} \backslash T_{a}$. Then it holds $\int_{T} f^{\prime}(s) d s=$ $y_{i} \in \mathcal{C}^{\mathrm{P}}(q, c)_{i}$. Moreover $\lambda(T)=q_{i}$, so $T \in \mathcal{Q}\left(\mathcal{C}^{\mathrm{P}}, S, i\right)$. Let $R_{T}:=\left(q, f_{T}\right)$. By $y \in \mathcal{C}^{\mathrm{P}}(q, f)$ it holds $y(Q \cup\{i\}) \geq f_{R}^{\mathrm{P}}(q(Q \cup\{i\}))$, so

$$
\begin{aligned}
y(Q) & \geq f_{R}^{\mathrm{P}}(q(Q \cup\{i\}))-y_{i}= \\
& =\int_{T_{q(Q \cup\{i\})}} f^{\prime}(s) \mathrm{d} s-\int_{T} f^{\prime}(s) \mathrm{d} s=\int_{T_{q(Q \cup\{i\})} \backslash T} f^{\prime}(s) \mathrm{d} s \\
& \geq \inf \left\{\int_{U} f^{\prime}(s) \mathrm{d} s \mid U \in \mathcal{B}([0, q(S)] \backslash T), \lambda(U)=q(Q)\right\} \\
& =\inf \left\{\int_{U}\left(f_{T}^{\prime}\right)(s) \mathrm{d} s \mid U \in \mathcal{B}([0, q(S \backslash\{i\})]), \lambda(U)=q(Q)\right\} \\
& =\left(f_{T}\right)_{R_{T}}^{\mathrm{P}}(q(Q)) .
\end{aligned}
$$

By variation of $Q$ and the fact that $y(S \backslash\{i\})=f_{T}(q(S \backslash\{i\}))$ we conclude that $y_{S \backslash\{i\}} \in \mathcal{C}^{\mathrm{P}}\left(R_{T}\right)$.

Part (ii): Let $\Psi$ be a consistent solution. Then we need to show that $\Psi(R) \subseteq \mathcal{C}^{\mathrm{P}}(R)$ for all problems $R=(q, f) \in \mathcal{R}$. We will start with a proof for $R \in \mathcal{R}^{N}$ and a similar reasoning applies for arbitrary $R \in \mathcal{R}^{S}$. So assume $\Psi \in \mathcal{S}(\mathrm{CO}), R \in \mathcal{R}^{N}$, and $x \in \Psi(R) \subseteq \mathcal{I}^{\mathrm{P}}(R)$. Then it suffices to prove that for any $S \subseteq N, x(S) \leq f_{R}^{\mathrm{P}}(q(S))$. By consistency it holds that there is a $T_{1} \in \mathcal{Q}(\Psi, R,\{1\})$ such that $x_{N \backslash\{i\}} \in \Psi\left(q_{N \backslash\{1\}}, f_{T_{1}}\right)$. Put $R^{1}=\left(q, f_{T_{1}}\right)$, then by consistency there is a $T_{2} \in \mathcal{Q}\left(\Psi, R^{1},\{2\}\right)$ such that $x_{N \backslash\{1,2\}} \in \Psi\left(q_{N \backslash\{1,2\}},\left(f_{T_{1}}\right)_{T_{2}}\right)$.Put $R^{2}=\left(q_{N \backslash\{1,2\}},\left(f_{T_{1}}\right)_{T_{2}}\right)$. This procedure may now be repeated for the agents $3,4, \ldots, n$. In this way we obtain profit sharing problems $R^{0}, R^{1}, \ldots, R^{n-1}$, and $R^{n}$, such that $R^{0}=R$, and for $i \in N, R^{i}=\left(q_{N \backslash\{1,2, \ldots, i\}}, f^{i}\right) \in \mathcal{R}^{N \backslash\{1,2, \ldots, i\}}$ is such that $f^{i}=f_{T_{i-1}}^{i-1}$ for 
some $T_{i-1} \in \mathcal{Q}\left(\Psi, R^{i-1},\{i\}\right)$ with the property that $x_{N \backslash\{1,2, \ldots, i\}} \in \Psi\left(R^{i}\right)$. In particular by definition of a reduction it holds for all $i \in N$

$$
x_{i}=\int_{T_{i}}\left(f^{i}\right)^{\prime}(s) d s
$$

Define increasing bijections $g_{i}:\left[0, q(N \backslash\{1,2, \ldots, i-1\}] \rightarrow\left[0, q(N \backslash\{1,2, \ldots, i-1\})-\lambda\left(T_{i}\right)\right]\right.$ by

$$
g_{i}(s)=\lambda\left([0, s] \backslash T_{i}\right) \text { for all } s \in[0, q(N \backslash\{1,2, \ldots, i-1\})] .
$$

Note that

$$
\lambda\left(g_{i}(U)\right)=\lambda(U) \text { for all } i \in N
$$

Next, define

$$
T_{i}^{*}:=g_{1}^{-1} \circ g_{2}^{-1} \circ \ldots \circ g_{i-1}^{-1}\left(T_{i}\right) \text { for } i \in N .
$$

The collection $\left\{T_{1}^{*}, T_{2}^{*}, \ldots, T_{n}^{*}\right\}$ satisfies the following three properties:

a) $\lambda\left(T_{i}^{*} \cap T_{j}^{*}\right)=0$ for all $i \neq, j$, since $g_{\ell}$ 's are bijections,

b) $\lambda\left(T_{i}^{*}\right)=q_{i}$ for all $i \in N$, by (26), and

c) $x_{i}=\int_{T_{i}^{*}} f^{\prime}(s) \mathrm{d} s$ for all $i \in N$, by $(25)$.

Hence

$$
x(S)=\sum_{i \in S} \int_{T_{i}^{*}} f^{\prime}(s) \mathrm{d} s \geq \inf \left\{\int_{T} f^{\prime}(s) \mathrm{d} s \mid T \in \mathcal{B}([0, q(N)]) ; \lambda(T)=q(S)\right\}=f_{R}^{\mathrm{P}}(q(S)) .
$$

